\title{
LOGICAL TOPOLOGY DESIGN FOR IP REROUTING: ASONS VERSUS STATIC OTNS
}

\author{
Adelbert Groebbens ${ }^{*}$, Didier Colle ${ }^{*}$, Sophie De Maesschalck ${ }^{*}$, Bart Puype ${ }^{*}$, Kris \\ Steenhaut ${ }^{0}$, Mario Pickavet ${ }^{*}$, Ann Nowé ${ }^{0}$, Piet Demeester ${ }^{*}$ \\ *Dept. of Information Technology, Ghent University - IBBT - IMEC, \\ Gaston Crommenlaan 8, 9050 Gent (Belgium) \\ Phone: +3293314900 \\ Fax: +3293314899 \\ Email: \{adelbert.groebbens, didier.colle, sophie.demaesschalck, bart.puype, mario.pickavet, \\ piet.demeester\}@intec.ugent.be
}

${ }^{o}$ Vrije Universiteit Brussel - TW - ETRO, COMO

Pleinlaan 2, 1050 Brussel (Belgium)

Phone:+3226292985

Fax:+32 26292870

Email: \{ksteenha, asnowe\}@info.vub.ac.be

Contact author: Mario Pickavet

\begin{abstract}
IP based backbone networks are gradually moving to a network model consisting of high-speed routers that are flexibly interconnected by a mesh of light paths set up by an Optical Transport Network (OTN) that consists of WDM links and Optical Cross-Connects (OXCs). In such a model, the Generalized MPLS (GMPLS) protocol suite could provide the IP centric control plane component that will be used to deliver rapid and dynamic circuit provisioning of end-to-end optical light paths between the routers. This is called an Automatically Switched Optical (transport) Network (ASON). An ASON enables reconfiguration of the logical IP topology by setting up and tearing down light paths. This allows to up- or downgrade link capacities during a router failure to the capacities needed by the new routing of the affected traffic. This way of making the IP layer survivable against (single) IP router failures can be cost effective, as capacity to the IP layer can be provided flexibly when necessary. We present and investigate a logical topology optimization problem that minimizes the total amount or cost of the needed resources (interfaces, wavelengths, WDM line-systems, amplifiers,...) in both the IP and optical layer. A novel optimization aspect in this problem is the possibility, as a result of the ASON, to reuse the physical resources (like interface cards and WDM line-systems) over the different network states (the failure free and all the router failure scenarios). We devised a simple optimization strategy in order to investigate the cost of the ASON approach and compare it with other schemes that survive single router failures.
\end{abstract}

Keywords: IP-over-WDM, Optical Transport Networks, Automatic Switched Optical Networks, IP topology reconfiguration, Survivability 


\section{Introduction and related work}

\subsection{Benefit of an ASON versus a static OTN for IP recovery}

Up till now, Optical Transport Networks (OTNs), based on Wavelength Division Multiplexing (WDM) technology and the deployment of Optical Cross-Connects (OXC), are statically configured by the network operator through the Network Management System (NMS). With this system, manual interventions are still needed to satisfy certain client requests such as connection set-up or tear-down, and this result in a long provisioning time. In order to be able to cope with this dynamic Internet traffic, current standardisation and research efforts are investigating the introduction of more intelligence and autonomy into the OTN in the form of a separated and distributed control plane. This type of optical network is denoted as an Automatic Switched Optical Network (ASON) [1, 3, 8] or sometimes also as an Intelligent Optical Network (ION). By introducing such a distributed control scheme, the client no longer has to contact the transport network operator to request the set-up or tear-down of a connection, but talks directly to the transport network access - through the User-Network Interface (UNI) - to trigger these requests. Connections established in this manner are called switched connections (SCs). In this way, any manual action from the network operator's side is omitted, resulting in increased network flexibility and dynamics.

In this paper, we focus on IP as the client layer for the optical transport network and call this the multi-layer "IP-over-optical" network scenario. In an IP-overoptical network, the recovery against core router failures is important, because current core IP routers are not reliable enough to assume no router failures (see [2] and [3]). What is more, the recovery of the failure of the upper layer nodes (routers in IP-over-optical) must be dealt with by the upper layer even in the context of a multi-layer recovery strategy. Surprisingly, the above mentioned network flexibility and dynamics of ASONs, allows us to appropriately reconfigure the logical IP layer topology in case of a router failure. This is achieved by adjusting the switched connections in the optical transport network. Such reconfigurations could involve either the increase or reduction of the capacity on a certain link, or also the addition or removal of an IP link (also called adjacency). 
Let us carefully explain this: In the concise example of Fig. 1, the light paths that are needed before and after a router failure (router $b$ ) are shown. The light paths provide the links in the IP layer and these links support the IP packet flow from router $a$ to router $c$. Such a scheme for recovery against a single router failure is called "IP rerouting over ASON" in this paper. Note that when the failure of router $b$ occurs, the two existing light paths ( $\mathrm{a}-\mathrm{b}$ and $\mathrm{b}-\mathrm{c}$, called the working light paths) are torn down and then the new requested light path (called the backup light path) is set up. We use the term 'backup light path', because it is only used for supporting backup IP flows after a router failure. This should not be confused with light paths that are protecting other light paths in the optical layer $\neg-$ this paper does not focus on protection in the optical layer. The 'working light paths' carry nominal working IP flows. They could occasionally be groomed with extra backup IP flows that are activated after a router failure.
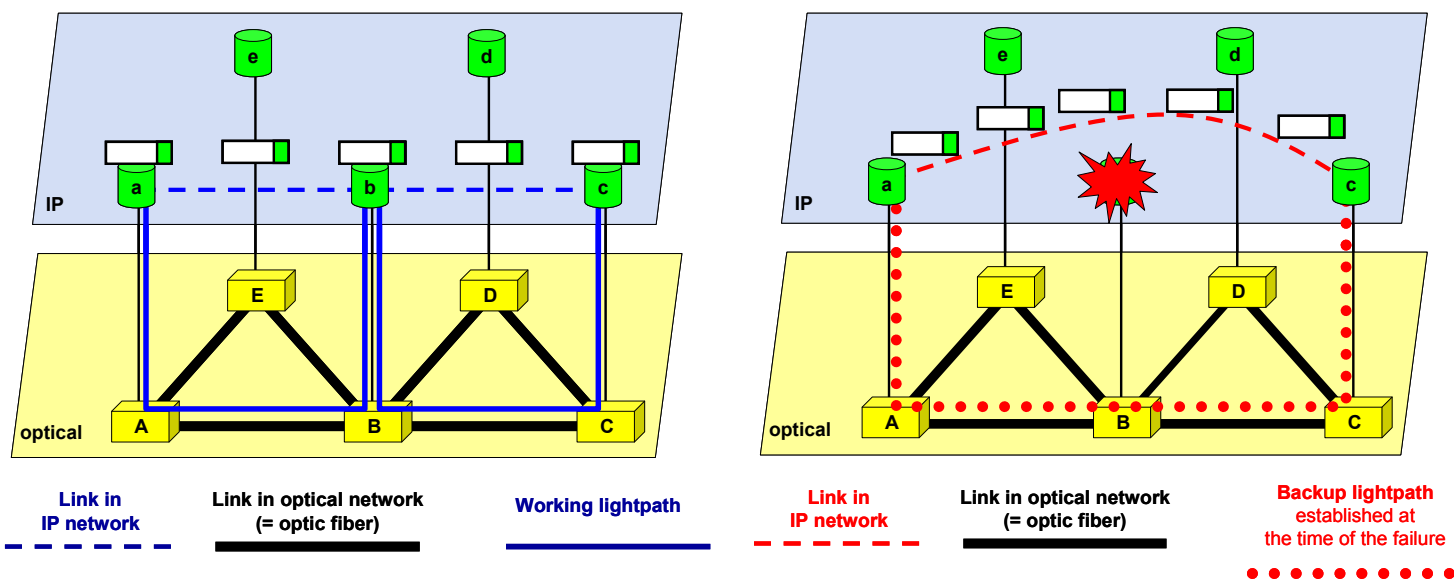

Fig. 1 IP rerouting over ASON: failure free (left) and failure of router $b$ (right).

Now, let us contrast this with the current practice of a statically configured OTN (also called a "static OTN") that is managed by operators using the NMS. The recovery deployed in such a static OTN is depicted in Fig. 2. Again, the same IP traffic flow from $a$ to $c$ is depicted - supported by the same two working light paths. But now extra backup light paths that are only supporting some backup IP flows need to be explicitly present and set-up in advance ("long" before the failure). The reason is that the manual intervention by the operator $\neg-$ that is necessary for setting up or tearing down light paths in a static OTN - would be too slow for recovery purposes in case of a router failure. This scheme for recovery against a single router failure is called "IP rerouting over static OTN" in this paper. 

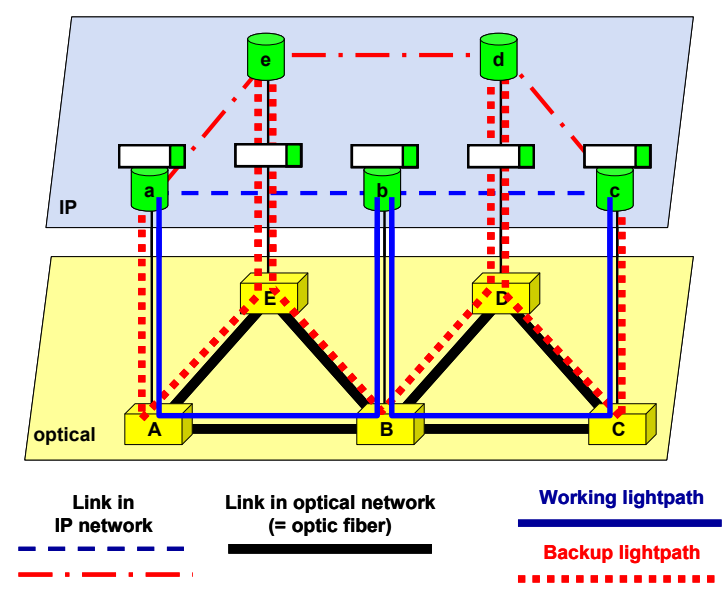

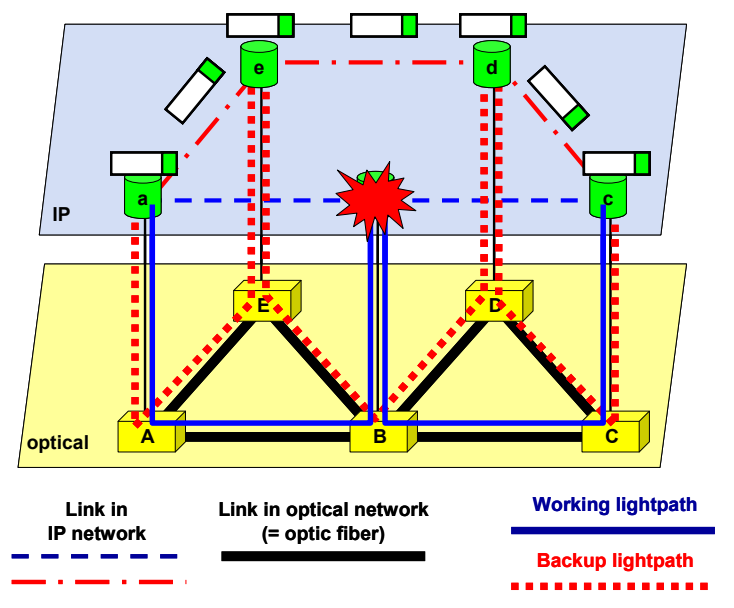

Fig. 2 IP rerouting over static OTN: failure free (left) and failure of router $b$ (right).

Thus, we come to the core comparison to be made in this paper. In both figures above, resources (in terms of interface cards, transponders, OXC ports, ...) are needed for the working light paths, which are most of the time active. But IP rerouting over ASON poses more opportunities for reuse of resources than IP rerouting over static OTN.

For example, in IP rerouting over ASON, it is possible for the backup light paths to reuse resources that were used previously by the working light paths that are affected by the failure. This is illustrated in Fig. 1: the IP interface card at router $a$ for working light path $\mathrm{a}-\mathrm{b}$ can be reused to establish the backup light path a-c. Another more complex example of reuse in the IP rerouting over ASON scheme not illustrated by the figures above - is to use an interface card at a source router for a backup light path that will already be used for another backup light path to a different next hop and that is protecting for a different router failure. In that case, the latter light path exist for recovery against a router failure that the former light path does not need recover for, because the working IP flows pass through different routers. This is illustrated in Fig. 3: an example with two IP flows is given. The configuration for the IP rerouting over static OTN scheme is shown. The working light paths for the flow $I$ and $I I$ are left out with the purpose of not overburdening the figure. There a just four working light paths of one optical hop: A-B, B-C for flow I and E-D and D-C for flow II. The path of the IP backup flow for the flow $I$ is a-e-d-c. For the second one, it is e-b-c. The light paths (and the links they form) related to the second flow are drawn on top of a shade. In the figure, which depicts the configuration for the IP rerouting over static OTN scheme, the light paths e-b and e-d would lead to a requirement of at least two 
interfaces at router $e$. For the IP rerouting over ASON scheme, this would only be one interface. The reason is that backup light path e-b is protecting working flow II against a failure of router $d$ and that backup light path e-d is protecting working flow $I$ against a failure of router $b$.

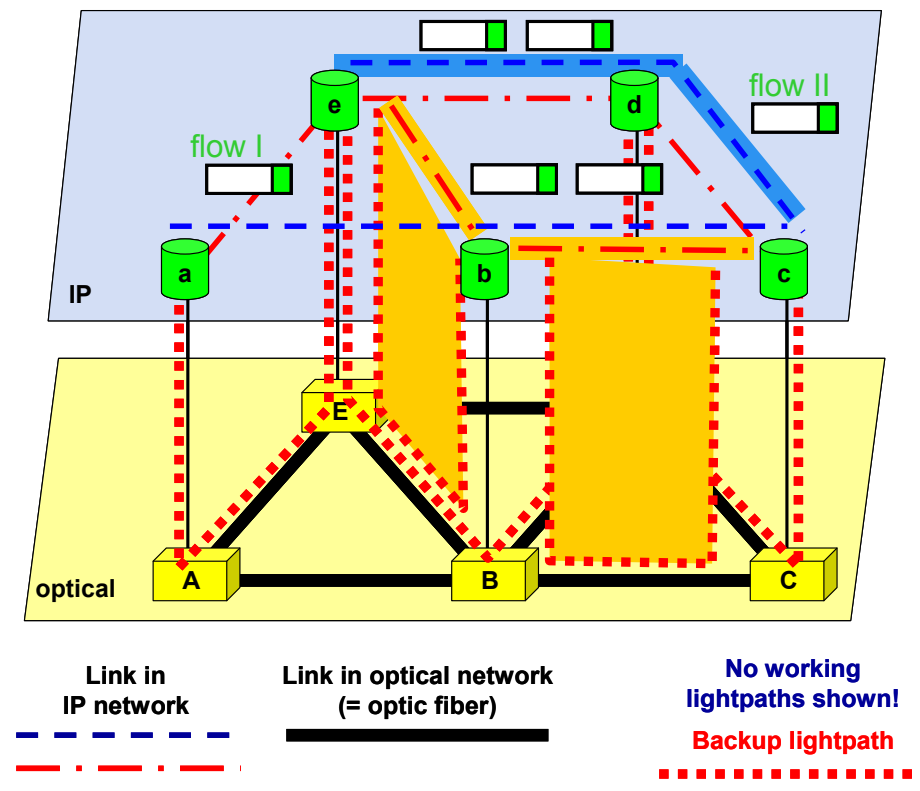

Fig. 3 Configuration example for IP rerouting over static OTN that gives rise to a more complex form of reuse in IP rerouting over ASON.

At the same time there exists corresponding reuse for wavelengths (instantiated by transponders and OXC ports) at the optical links in IP rerouting over ASON. This can also be demonstrated by means of Fig. 3. In IP rerouting over ASON, the backup light paths e-d and e-b could for example reuse the same wavelength at the line-system E-B. Note from above that the route of the working light path e-d for the first hop of flow II is along the optical path E-D, but the backup light paths e-b and $b-d$ are going via $\mathrm{OXC} \mathrm{B}$. This was chosen purely to illustrate that the sharing can happen between backup light paths only. In IP rerouting over static OTN, two wavelengths would be necessary for the given configuration at E-B.

It is important to realize that the flexibility of ASON gives rise to a new form of reusability of resources. This form comes in addition to the well-known reuse from sharing backup bandwidth (BBW) protecting different risks or from the stub release of affected working paths (see Section 2.3). These well-known reuses are due to the packet based network characteristics of the IP layer.

In short, IP rerouting over ASON is supposed to be more effective in terms of capacity and cost than IP rerouting over static OTN. This was touched in [3] and a 
glimpse on the effect of the proper choice of the logical topology and the optimized path routing in order to obtain a cost-efficient solution was presented. The study in this paper continues this line of thought, and focuses on how to design an efficient logical topology for IP rerouting over ASON in order to achieve this cost-effectiveness for recovery against single router failures. The benefits of an ASON versus a static OTN for recovery against single router failures, while using an efficient logical topology will be quantified. For both schemes, the method of recovery in the IP layer will be the standard shortest-path routing (and rerouting) that is present in the currently deployed link state routing protocols. The rerouting is done by updating the routing tables from the moment that a change (e.g., a failure) in the topology (status) has been detected. This "IP rerouting" will be explained from a capacity viewpoint in Section 2.3. This paper will focus on the question "does logical topology optimization make sense when using shortest hop IP (re)routing ?"

\subsection{Outline of the rest of the paper}

In Section 2, we will give more details on the IP rerouting over ASON scheme. First, we will include a detailed description of the logical topology optimization problem for minimizing installed resources when using this scheme. At the same time, we will explain the used model (traffic, network, layers, routing, rerouting, costs and components, objective, ...) and the overall assumptions. In Section 3, we will extend and adapt this model to include (1) IP rerouting over static OTN and (2) a capacity benchmark considering only the grooming of the working traffic. Extension (2) will be purely used as a base line for the comparison. Next, we will consider the optimization approaches we devised for this (these) problem(s). Section 4 will consider different logical topology designs and the optimization algorithm to obtain them. In Section 5, we will discuss several case studies and results obtained. In Section 6, we round up with conclusions.

\subsection{Main contributions}

The main contributions of this paper are the following:

- The use of ASONs for recovery is presented in detail. 
- A logical topology problem for minimizing capacity installation (or its cost) in IP-over-ASONs that are survivable against router failures is introduced (with a novel resource sharing aspect).

- Quantitative results from case studies and overall conclusions and insights around this problem are presented (preliminary results were presented in [11] and [12]).

- The developed tool and approach for optimizing the logical topology (with the restriction of shortest-path routing) in case of an IP-over-optical network is presented.

- A survey of related work and optimization problems is given.

\section{Cost effective IP rerouting over ASON: model and optimization problem}

In this section, we will explain in more detail the IP rerouting over ASON model. We will state the assumptions made in our nodes and network model, the traffic model, the routing and rerouting model and the dimensioning model. In order to fully exploit the advantage in resource requirements of IP rerouting over ASON, compared to IP rerouting over static OTN, it is necessary to look at the dimensioning problems minimizing the resources. This is the second subject of this section. We will describe in particular the two investigated objectives (cost models) and we will conclude with some remarks and related work about this optimization problem.

\subsection{Node, network, multi-layer and resource models}

Most aspects of our model are depicted in Fig. 4. We have an IP layer with routers on top of an optical layer with optical cross-connects (OXCs). The OXCs are OEO optical switches, enabling wavelength conversion via OEO. In the figure, an example is given of one uni-directional light path that is set up between source router $a$ and destination router $b$. Our whole model is uni-directional.

The figure displays a uni-directional transmitting IP interface at router $a$, that is sending out at a grey wavelength $\lambda^{*}$. A receiver port (rx) at OXC takes the signal and then it is switched to a transmitter port (tx). Subsequently, the signal goes through a tx transponder, which is converting the signal from a grey to a colored 
wavelength $\lambda_{\mathrm{i}}$. Next, the colored wavelengths (lambdas) are multiplexed into a fibre. Along the fibre, the signal is amplified every K kilometres. Finally, by demultiplexing and converting from $\lambda_{\mathrm{i}}$ to a $\lambda^{*}$, the signal is delivered through the $\mathrm{OXC} \mathrm{B}$ at the ingoing interface card at router $b$.

In our network, all routers are core routers and are subject to failure. All will have an OXC below them, which can be connected to multiple WDM line-systems. The associated IP router, OXC and line-systems are collocated in the same building. We assume that the interconnection between the $\mathrm{OXC}$ and the router does not fail. We also take for granted that one IP router and one OXC will be present at each location (we do not take into account the additional costs if more equipment boxes are needed in case of very high interface or port requirements).

We only consider a given fixed bit rate capacity $\mathrm{C}$ for the IP interfaces. Likewise, only a given fixed bit rate capacity of the wavelengths/light paths is assumed. This bit rate of the IP interfaces is of course the same (thus C).

WDM line-systems correspond with the (de-)multiplexer, transponders and fibre pair with the amplifiers. We assume that a uni-directional transponder can be added to a multiplexer (or demultiplexer) on an as needed basis (one by one). Likewise, the uni-directional OXC ports can be added one by one to the optical switch. The multiplexers include a post-amplifier (a booster) and the demultiplexers a pre-amplifier.

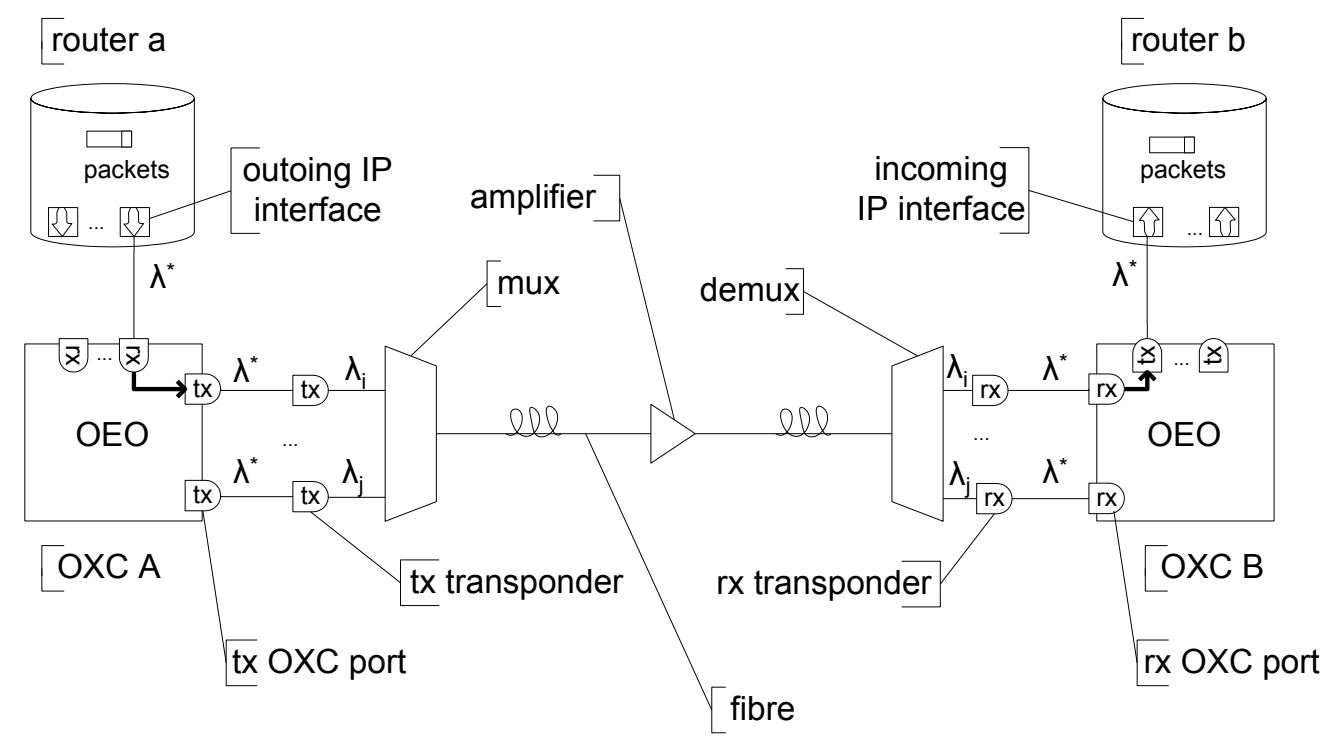

Fig 4 IP router and OXC model with an example of a uni-directional light path from router $a$ to $b$.

Fig. 4 also shows the cross-connections made in the OXC for a light path that is set-up from router $a$ to $b$. They form a light path consisting of a single wavelength 
on the depicted line-system. In the $\mathrm{OXC}$, the conversion "from optical to electrical and from electrical-optical" (OEO) that is carried out enables the translation of the original colour of a wavelength into a new and possibly different one.

If a light path is going to transit multiple OXCs, then multiple wavelengths on different line-systems could be occupied and made 'alive' along the path. If a lot of wavelengths are needed between a source-destination (S-D) OXC pair, then it is necessary to install multiple line-systems. We consider a given fixed capacity W for the WDM line-systems in terms of wavelengths.

\subsection{Traffic model}

The traffic demand for the network consists out of IP traffic flows of a certain capacity from every source to every destination IP router. The flows are unidirectional [8] and the traffic matrix can be asymmetric [8]: flows from A to B and B to A bear no constraints with respect to each other. All traffic for a sourcedestination (S-D) router pair is aggregated into one IP traffic flow. The bit rate of a given S-D traffic flow can be less or greater than the bit rate $\mathrm{C}$ that is supported by an optical wavelength channel. The aggregated IP flows are not bound to be in just one optical light path: they can be split over more than one. The bandwidth of the traffic flows will be generated based on realistic traffic matrices (see Section $5)$.

\subsection{Routing and rerouting model: shortest paths}

In this section we explain the routing and rerouting model that is used throughout the whole paper (except for TE and bifurcated grooming introduced in Section 3.3). First, the routing and rerouting in the IP layer is discussed, then the optical layer follows. It is important to stress that these models apply to both survivable schemes (IP rerouting over ASON; IP rerouting over static OTN) and the grooming benchmarks (see later).

\subsubsection{At the IP layer}

As already explained, in our network, all routers are core routers and are subject to failure. We assume that our network dimensioning and planning is restricted to fully cover single router failures (as full coverage of multiple failures would probably generate not enough revenue with respect to the costs of it). 
The IP layer employs shortest-path routing as provided by an Interior Gateway Protocol (IGP) like the well known link state based routing protocol "Open Shortest Path First" (OSPF). OSPF is also used when a router fails: new shortestpaths will be followed by the traffic flows, surrounding the failed router. This is sometimes called "IGP convergence" or "IP restoration".

Our routing and rerouting model that will be used and can be applied to all our schemes is illustrated in Fig. 5. In that figure, we have shown a logical IP topology with its links and their OSPF metric (= the weights). Next, there is a traffic flow from router $a$ to router $g$. We explain the figure drawings from left to right and from top to bottom: first, the failure free scenario (FFS) is shown, also called the normal or nominal scenario. Then the failures of the intermediate routers $c, d$ and $f$ are shown one after another. Because the IP flow transits these routers, it needs to be rerouted every time along a new shortest-path. In the penultimate drawing in the figure, it is shown that router failure of the source or destination router of the IP flow does not need to be rerouted but leads to traffic loss.

This sort of rerouting is sometimes called "failure-dependent backup routing" because there are multiple backup paths with one backup per particular failure. Let us assume that the bandwidth of the IP flows is $80 \%$ of C (i.e., the bit rate of a light path unit). Then, in the last drawing, the worst case bandwidth needs between the routers for the routing and rerouting of the flow is derived. It demonstrates that there is a bandwidth of nine light paths units needed if the given topology and OSPF metric of the example is used. This is regardless of the used survivable scheme: in IP rerouting over ASON this bandwidth could be provided on demand, in IP rerouting over static OTN this bandwidth would needed to be installed in advance. (Note that this topology would be a very inefficient choice for supporting the given flow of the example: a direct link from a to $g$ would be much more efficient - again regardless of the used scheme). Thus, the last drawing of Fig. 5 demonstrates that the multiple backup paths share bandwidth. They share capacity with other backup paths and with the working path (stub release). This well-known form of bandwidth sharing is purely due to the fact that IP flows consist of packets. 

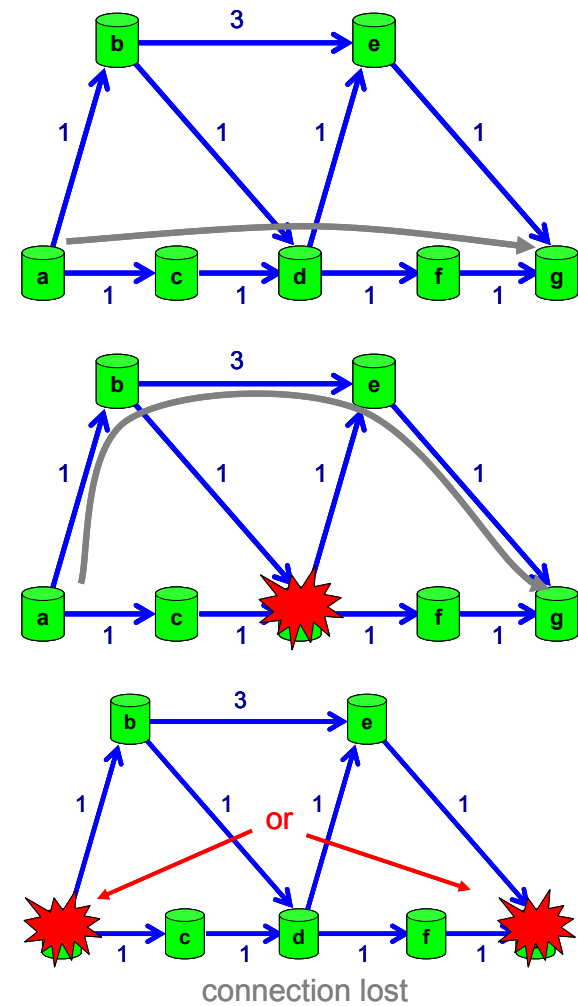
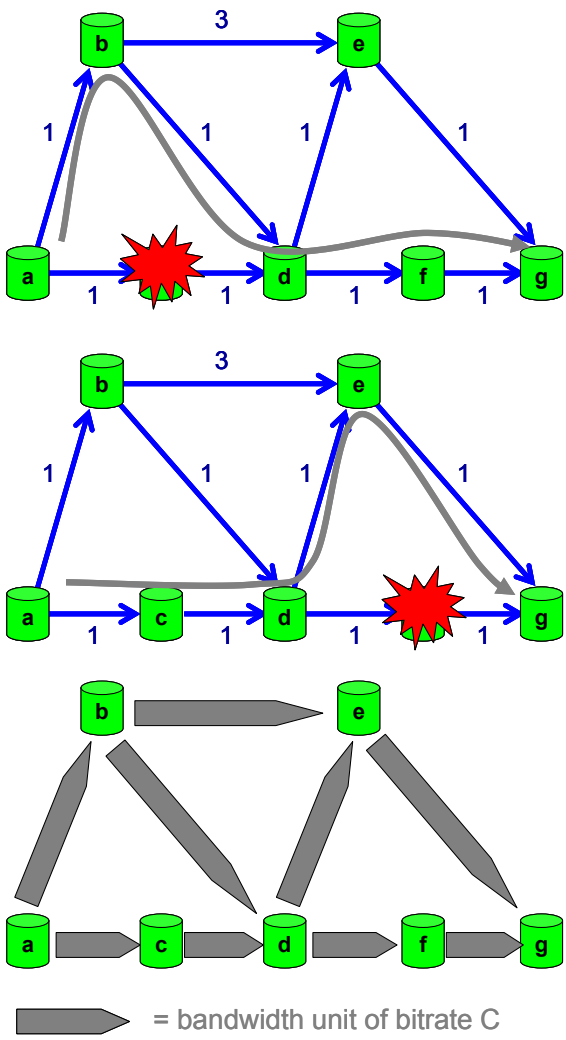

Fig 5 Bandwidth sharing example among a working IP flow and its multiple backup IP flows (example with one flow having a capacity of $80 \%$ of the bit rate unit of a light path).

In a normal traffic demand, there are multiple working IP flows, and then it is possible that multiple backup paths from distinct working IP flows share bandwidth, as their working flows are not simultaneously affected by a given single router failure. Again, this follows naturally from the fact that IP flows consist out of packets and has nothing to do with the survivable scheme (IP rerouting over ASON and IP rerouting over static OTN). If two working flows are coincident along their whole path, then their respective overlapping backup flows would be always simultaneously activated. In any other situation the two backup flows could share some bandwidth somewhere at their overlapping links. The effect on the bandwidth needs (in terms of light path units) of this kind of bandwidth sharing among backup flows is shown in Fig. 6. The sharing is illustrated by the fact that there is only one bit rate unit $(C)$ needed at link from $d$ to e. Again, this has nothing to do with our two considered survivable schemes. Our schemes only differentiate the point in time of provisioning enough bandwidth by setting up light paths between the routers at the endpoints of the link from $d$ to e. 

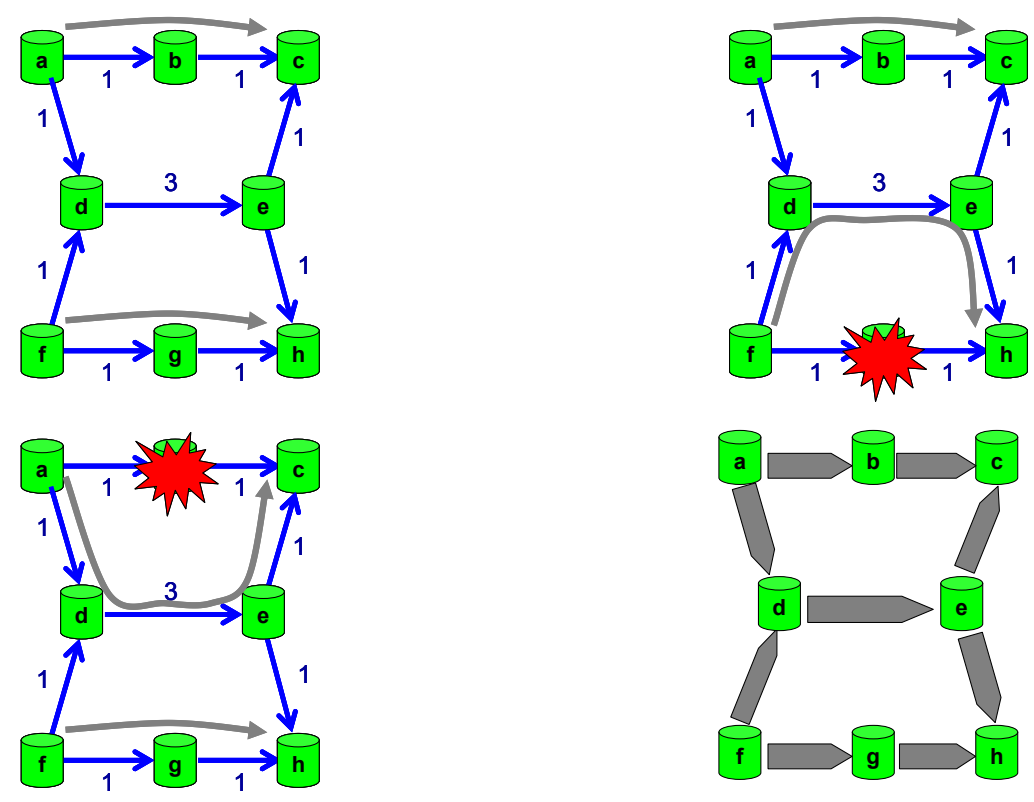

= bandwidth unit of bitrate $\mathrm{C}$

Fig 6 Bandwidth sharing example among backup flows of different working IP flows (example with two IP flows, each having a capacity of $80 \%$ of C).

Note that this routing and rerouting model implies that the logical topology must provide an alternative flow for every S-D pair of the (non-zero) traffic demand in case there would be a router failure. As a result, the topology will be at least biconnected between these S-D pairs. As such, the traffic demand imposes connectivity constraints on the logical topology.

Also of importance is our choice to use only a single shortest path among the equal-cost shortest paths (see Fig. 5, a-b-d-f-g and a-c-d-e-g were other shortest paths from a to $\mathrm{g}$ ) for every S-D pair (= aggregated S-D IP flow). The single shortest path is randomly selected among the equal-cost shortest paths.

Throughout this paper, all weights for use in shortest path computations are set to one. This is called "shortest-hop routing". It has the advantage that it is easy deployable for an operator. Even more, router implementations could be optimized (or made more scalable) in providing a shortest-hop routing. In theory, our approach (see below) supports any fixed set of weights: i.e. one predetermined weight for each edge from the given possible edges of the logical topology. Among all the possibilities of fixed sets weights, the "all weights one" option is quite a reasonable one since every single path used in the shortest-path routing tries to consume as few resources as possible. 
Another advantage of shortest-hop routing (or shortest-path routing in general) is that it intensifies the locality of the extra required resources when rerouting the IP flows. It was demonstrated in [3] that locality in rerouting is a property that helps for an efficient resource dimensioning for IP rerouting over ASON (see Section 2.4). We admit that a single shortest-hop routed path does not provides the best load balancing of the flows over the network, although the choice of the topology structure (the presences of edges) can partly relief this.

IP rerouting over ASON (see Section 2) implies that some light paths need to be set-up for coping with bandwidth insufficiency over the logical link along the new recovery path - when rerouting the affected IP flow. On the other hand, the light paths that are left unequipped after the rerouting of the affected IP flows will be torn down. We will assume that the tearing-down processes always happen before the setting-up processes (this is called "break-before-make").

The setting-up and tearing-down events will only take place in IP rerouting over ASON. For IP rerouting over static OTN (see Section 3.1) enough bandwidth capacity will be available by design. As a result, the tearing-down of light paths will not happen. In fact it can not happen due to the static nature of the OTN. Note that in the figures above and in our schemes below, a single logical topology structure will be used for the shortest-path computations in all network states. Thus it will be used for the failure free routing and the rerouting after every single router failure. This leads to the locality mentioned before. Furthermore, the single logical topology make sure that only the affected IP flows will be rerouted in case of a single router failure. The path of the unaffected IP flows will remain a shortest-path and consequently its routing will not change. This is a useful property in relation to quality of service (QoS): unaffected working paths will not be interrupted.

\subsubsection{At the OTN layer}

Every S-D router pair will be associated with a shortest path between its underlying OXCs in the optical layer. As such, all light paths between an S-D pair will follow the same route. We provide no survivability in the optical layer (against fibre cuts or OXC node failures for example) as the comparison between ASONs and static OTNs for single router failure recovery is our main focus. Survivability in the optical layer can be an extension to our model. Again, only a single shortest path will be chosen among the equal-cost shortest paths. The 
shortest-path metric is based on length of the optical links. In this way, we try to reduce the consumption of optical node resources and fibre amplifiers for example. In short, we will have only working light paths at the OTN layer.

\subsection{Dimensioning: reuse of resources enabled by an ASON}

Fig. 7 is the key figure of the paper as it is focusing on the dimensioning of IP rerouting over ASON. Let us first describe the left part of the figure: the failure free scenario (FFS). It shows the IP traffic demand and four conceptual layers, involving: the IP layer (with round routers); the optical layer (with rectangular OXCs); their routing; and their capacity dimensioning. The two network layers are both shown in a grey plane. The traffic demand shows five IP traffic flows, that are assumed to claim half of the capacity of a light path (interface card, wavelength channel) leading to a total demand of $2.5 \times \mathrm{C}$. In the top layer, below the failure free traffic demand, the routers $(\mathrm{Ra}, \mathrm{Rb}, \mathrm{Rc}$, and $\mathrm{Rd})$ are interconnected by logical uni-directional adjacencies. A directed adjacency represents the possibility to route traffic from its source to its destination end router. The traffic routes are also shown on that same top layer in thicker arrowed lines. They follow shortest paths with respect to the given distance metric (i.e., the numbers present in grey plane at the top in the figure) at the adjacencies.

From the routing, one can compute the necessary amount of uni-directional light paths between every source-destination (S-D) router pair, as well as the number of outgoing (arrow up in the figure) and incoming (arrow down in the figure) interfaces needed at each router. The reader can trace a light path by following the arrow up (the outgoing interface), the arrow along the directed light path and the arrow down (the incoming interface). Every active light path needs one outgoing and one incoming interface. As told before, the capacity of a light path and an IP interface is given and predetermined for the whole network, let us say fixed to C. The light paths are shown on the second layer from above. Note that an adjacency between routers gets translated into zero, one or more light paths. This depends on the routing and the capacity demand value of the traffic flows.

These light paths imply traffic to be served by the optical layer. On the next layer (the third from above), the physical topology with its physical adjacencies (i.e., spans or fibre strands) and OXCs is shown. The uni-directional light paths also follow shortest-paths drawn in thicker lines, with respect to the shortest-path 
metric between the OXCs. The shortest-path metric will often be the fibre length of the optical adjacency. The fibre lengths are shown at the link in the bottom grey layer.

From the routing of the light paths, the number of wavelengths needed in the linesystems can be determined and the necessary amount of uni-directional linesystems at every optical link can be computed. The dimensioned line-systems are shown at the bottom layer in the figure. The capacity of a WDM line-system is also given for the whole network and fixed to W. Note again that an adjacency in the optical layer between two OXCs will lead to zero, one or more line-systems from its source to its destination $\mathrm{OXC}$.

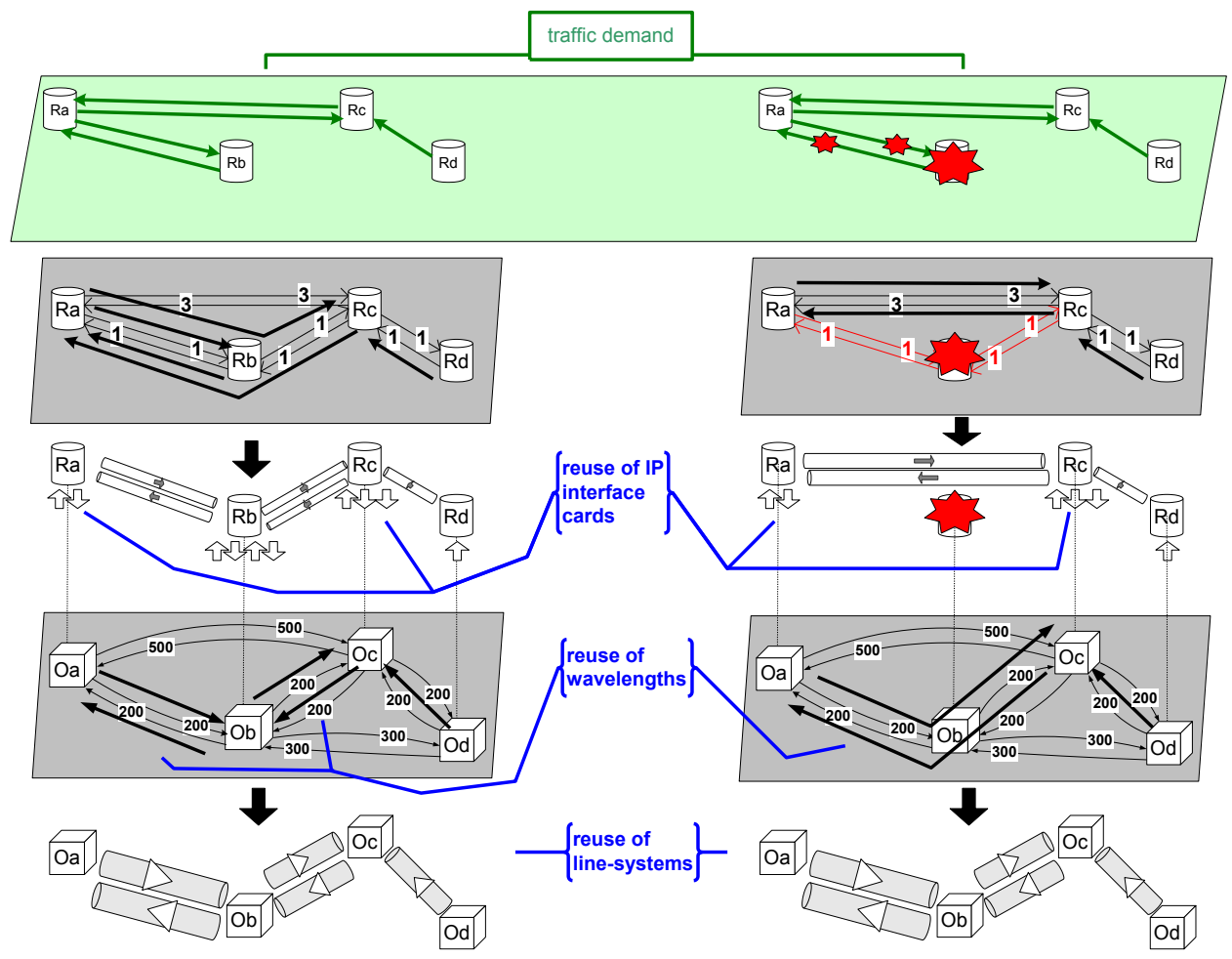

Fig 7 Detailed dimensioning example for the IP rerouting over ASON model: failure free scenario (left) and router $\mathrm{Rb}$ failure scenario (right).

From this of information, the rest of the capacity dimensioning can take-off: The line-systems determine the number of uni-directional multiplexers and demultiplexers. The distance of the adjacencies between OXCs determines the number of uni-directional amplifiers to be installed along the uni-directional fibre that is needed for a line-system. The wavelengths determine the number of unidirectional OXC ports and WDM transponders. Light paths are indeed virtual resources that are physically provided by interface cards, ports, equipped wavelengths, etc. 
Next, we turn our attention to the part on the right of Fig. 7: this figure part is specific for IP rerouting over ASON. It presents a single router failure scenario, namely that of router $\mathrm{Rb}$. The failure means that the routers' adjacencies are not available for use: all the interfaces needed for the light paths to and from the router are failing. It also means that the IP traffic demand only consists out of three S-D flows. Now, the routing of the IP traffic flows needs to go over alternative shortest paths. As a result, the whole dimensioning (interfaces, wavelengths, line-systems) changes.

Considering IP rerouting over ASON, the recovery is successful: an ASON enables us to set up or tear down light paths, when a router failure occurs. Consequently for the given router failure scenario (RFS), as indicated in Fig. 7, we are able to reuse the existing IP router interfaces and wavelengths and the linesystems of the failure free scenario (FFS). A concrete example is that the IP interface that was connecting from router $\mathrm{Ra}$ to router $\mathrm{Rb}$ in the FFS, is now connecting from router $\mathrm{Ra}$ to router $\mathrm{Rc}$ after the occurrence of the router failure of $\mathrm{Rb}$ in the RFS of $\mathrm{Rb}$. As a result of this interface reuse, there are only two unidirectional interfaces needed at router $\mathrm{Ra}$, and not four. The same idea applies to the reuse of wavelengths (and their supporting physical resources like OXC ports and transponders) and the line-systems.

This reuse is not limited to the depicted scenarios in Fig. 6. In general, in a network of $\mathrm{N}$ nodes (i.e., locations of an IP router plus an OXC), we have one failure free scenario (FFS) and N router failure scenarios RFS(i) - one for every router $i=1 \ldots \mathrm{N}$. Every RFS $(i)$ can reuse existing resources of the FFS, and also of another router failure scenario RFS(k). In the example of Fig. 7, only the router failure scenario RFS(Rb) is shown. In the other scenarios - RFS(Ra), RFS(Rc) and RFS(Rd) - there is no affected transit traffic in the routers. There is only traffic that is un-recoverably lost because the source or destination router is failing. As a result, the (capacity) dimensionings for these scenarios are consequently a subset of the FFS dimensioning.

Briefly we can state that the global dimensioning of the IP rerouting over ASON takes the explained reuse into account. The final result is called "the combined dimensioning". The combined dimensioning takes the maximum of the number of interfaces at an IP router and the number of wavelengths at a physical layer adjacency, over the FFS and all RFS( $i$ ) (for every router $i$ ). For Fig. 7, this leads to 
ten interface cards, five wavelengths and five line-systems to support the given traffic demand with recovery against single router failures.

At last note in Fig. 7 that the shortest-path routing in the OTN layer can be advantageous for IP rerouting over ASON: the shortest-path from Oa to Oc passes through $\mathrm{Ob}$, and as a result it coincides with a shortest path from $\mathrm{Oa}$ to $\mathrm{Ob}$ and a shortest path from $\mathrm{Ob}$ to $\mathrm{Oc}$.

\subsection{Optimization problem}

As our goal is to quantify the benefits in terms of resources when deploying an IPover-ASON for recovery, we are now ready to formalize the optimization problem, including the specifics considered in this paper.

\subsubsection{Inputs}

The inputs are: (1) The physical topology together with the shortest-path routing weights. The weights of the metric will be the $\mathrm{km}$ airline distances between the OXC node cities (corrected with some factor to take real fibre distances into account). A link of the physical topology determines if it is possible to install zero, one or more line-systems on it. (2) The IP traffic demand matrix. This matrix gives the bit rate (e.g., in Mbps) of the demanded flows from every source to every destination router. (3) The shortest-path routing weights for the logical topology. They are predetermined for every link in the full mesh between the router nodes (see Section 2.3.1).

\subsubsection{Variables}

The variables are: (1) The structure of the logical topology, i.e. we choose between which source and destination router we will have a directed edge. The number of (uni-directional) light paths along a directed edge is determined by the routing, and is not a variable as such. We call this variable the "logical topology design".

\subsubsection{Objective}

The objective is to find the logical topology design that gives the minimum combination of resources in order to cope with the failure free scenario (FFS) and all single router failure scenarios $(\operatorname{RFS}(i))$. The price of the combination can be 
expressed as an amount of a certain type(s) of resource(s) or a cost. This is called a "cost model". We have investigated two cost models.

\section{Complete IP plus optical layer cost model}

In this model, we associate a cost for every resource component as presented in the model of Section 2.1. For more details on the exact cost number we refer to [10]. In all our experiments, we consider the number of wavelengths on a WDM line-system to be $\mathrm{W}=40$. We have data for two options for the capacity of the router interfaces, $\mathrm{OXC}$ ports and transponders: $\mathrm{C}=2.5 \mathrm{Gbps}$ and $\mathrm{C}=10 \mathrm{Gbps}$ channels. Fibre dig costs are not included and the physical topology is given. This situation corresponds with the business model of a company that has already its fibre laying in the ground, but wants to build a new network consisting of IP routers over OXC nodes - applicable when there is an upgrade from WDM pointto-point (PTP) links to an optical transport network with OXCs and WDM links (see [1]). The company will still need installation of amplifiers along fibres it chooses to use for its line-systems.

\section{Interface amount model}

We also looked at a cost model that only takes into account the number of unidirectional router interface cards. This is a model for the case when an ISP company is leasing light paths and when light paths between two routers in the network cost more or less the same for every S-D router pair. It is also assumed in this case that light paths can be hired on-demand when they are needed: so that the reuse of wavelengths for IP rerouting over ASON can take place. Under these assumptions, the cost will be roughly proportional to the number of interfaces. This simplified model can be used to investigate the first-order benefits of IP rerouting of ASONs over static OTNs.

\subsubsection{Constraints}

The constraints were described in the previous sections: the node, network, multilayer and resource models (including the given type of resources), the traffic must be satisfied, the routing and rerouting model leading to topology connectivity constraints, the specifics of the capacity dimensioning for IP rerouting over ASON. 


\subsection{A note about this optimization problem}

Our problem "logical topology design for IP (routing and) rerouting over an ASON" incorporates the following algorithmic aspects:

- Traffic grooming: the grooming of IP flows into light paths (of capacity C) and the grooming of light paths into WDM line-systems (of capacity $\mathrm{W})$, due to the presence of the granularities/modularities $\mathrm{C}$ and $\mathrm{W}$. There is not yet an overwhelming amount of research around traffic grooming particular for meshed networks minimizing the cost of resources.

Reference [7] discusses interface card amount optimization in meshed networks. In [8], traffic grooming in an IP-over-optical with TE and bifurcated grooming was considered (see also Section 3.3). A model for traffic grooming using TE and bifurcated flows, taking various constraints into account, is presented in [9].

- Logical topology design (LTD): in fact, this is closely related to traffic grooming. In LTD problems, the available capacities at the topology links and the available topology links themselves are not given as inputs. Instead, the routing of the flows and the necessary links and capacities to support it need to be determined. For our design problem, the links need to be determined, but the routing is derived (based on the given routing metric). Although, there is a lot of work in LTD for IP-over-WDM, recovery in the IP or optical layer is rarely considered. More often the wavelength continuity constraints (Routing and Wavelength Assignment (RWA)) when there is no wavelength conversion is the main focus. A clear description with a mathematical formulation of LTD (using TE and bifurcated grooming, see Section 3.3) is given in [15]. The LTD problem is closely related to the Multi-Commodity Capacity Installation (MCCI) problem (see [21] and [8]).

- Optimization with the restriction of shortest-path routing: we consider the design of a network with the constraint of shortest-path routing (within the link weights being predetermined). A seminal paper on optimization of the routing using shortest-path weights is [16]. There the weights are optimized considering a given fixed topology and the objective is to minimize the maximum congestion on a link. Moreover, the IP flows are split among equal cost shortest-paths, as is often the case in papers on 
shortest-path weights optimization. In [17], a mathematical model for shortest-path routing is given. In [18] and [19], weight optimization taking into account link or router failure is considered. In [20], the general complexity of optimized routing that is constrained by shortest path requirements is emphasized. This justifies why we adopted a fixed set of OSPF weights as inputs.

- Survivable network design: we consider router node failures and as a consequence the network connectivity and capacity dimensioning of the logical topology has to allow the rerouting of the traffic flows.

- Jointly Optimized Spare and Working Capacity Design: for more information on this topic see [22]. In case of IP rerouting over ASON, we add a new kind of reuse (sharing) to the problem: for example, one kind is the reuse of the same router interface cards over different adjacencies.

- IP-over-optical multi-layer network design: both networks are taken into account due to our cost model and multi-layer effects are present due to the mechanics of IP rerouting over ASON.

\subsection{Related work}

The basic idea of this paper was first presented in a previous publication [3], but the optimization of logical topology was not considered. Preliminary results regarding our logical topology design problem are presented in [11] and [12]. The concepts of static and dynamic multi-layer survivability are nicely summarized in [1].

A strongly related problem, investigating the total IP plus optical layer cost, is described in [4]. The working paths are given and routed over a given working topology, while in our work they are incorporated in the optimization. The transit traffic of a failing IP router is rerouted over new IP links that are direct links between the "previous router-next router" pair as seen from the failing router transited by the affected flow. In our solution this direct link would be available for shortest-path routing in the failure free scenario. The automatic reuse of the capacity of the working path is similar to our strategy.

Reference [5] proposes an idea that is similar to IP rerouting over ASON, but reuse of interfaces is not mentioned. On the other hand, shared mesh restoration in the OTN layer is considered there. Some dimensioning computations seem to be 
incorrect since the granularity of the light paths is not taken into account and consequently the available optical restoration capacity is not always sufficient to cover all router failures.

Paper [6] refers to our work in [3], applying the same idea, but without giving quantitative results. The authors consider dual-homed access routers (i.e., access router connected to two core routers) and the reuse of cabling between the PoP offices. In case of a core router failure, new topology links to the second core router are set up from the access router. This strategy is different from our single logical topology (and metric) and shortest-path routing. Paper [2] combines the idea of papers [5] and [6]. Shared mesh restoration at the optical layer is considered. A single shared spare core router is considered. If the core router fails then its incident access routers will be connected with the single spare core router. The spare core router is shared among all router failures, a strategy similar to our reuse of resources in IP rerouting over ASON. In paper [2], smart but only a rough estimate of the spare capacity needs against router failures in the IP layer, are taken into account. The authors employ 50\% (or 70\%) utilisation in the light paths as a rule of the thumb to deal with IP spare capacity for router failures.

In [17] a problem with many common points was investigated, incorporating OSPF weights optimization, topology design, capacity design, survivability (diversification, etc.). But reuse in case of router failures was not considered.

\section{Two comparison schemes: (1) IP rerouting over static OTN and (2) grooming benchmarks}

The goal of this section is to look at the competing scheme for IP rerouting over ASON, namely IP rerouting over static OTN. Next, we also discuss our two grooming bench marks: shortest-path grooming and TE and bifurcated grooming. We emphasize that the grooming benchmarks are solely to get a feeling for the results. They represent design options that are non-survivable against single router failures, but give us insight in how much extra capacity is needed for providing survivability. 


\subsection{IP rerouting over static OTN}

Most aspects of the model for IP rerouting over static OTN are the same as for IP rerouting over ASON. For example the routing and rerouting model is the same, but as mentioned above in Section 2.3, the dimensioning of IP rerouting over static OTN must take into account enough spare capacity in the IP layer for rerouting purposes. There is no way of asking more resources from the optical layer at the moment of failure due to the static and permanent nature of the OTN. This means that all light paths used for recovery and their interface end-points, need to be present before any failure occurs.

We can make the same dimensioning exercise for the same demand of the example from Fig. 7. This is presented in Fig. 8 - where the traffic demand was omitted. Note the differences: the dotted lines of the backup IP flows will be used to replace the two working IP flows that are transiting router $\mathrm{Rb}$ when that router fails. This means that two new light paths are needed between $\mathrm{Ra}$ and $\mathrm{Rc}$. The light paths are routed in the optical layer. Because the line-systems can accommodate a fairly large number of $\mathrm{W}$ (uni-directional) wavelengths (e.g., $\mathrm{W}=$ 40 ), the same amount of line-systems is needed for IP rerouting over static OTN as in Fig. 7.

The dimensioning for the FFS and all RFS( $i$ )'s are present in the same figure for IP rerouting over static OTN, and therefore we also call Fig. 7 the "combined dimensioning". 


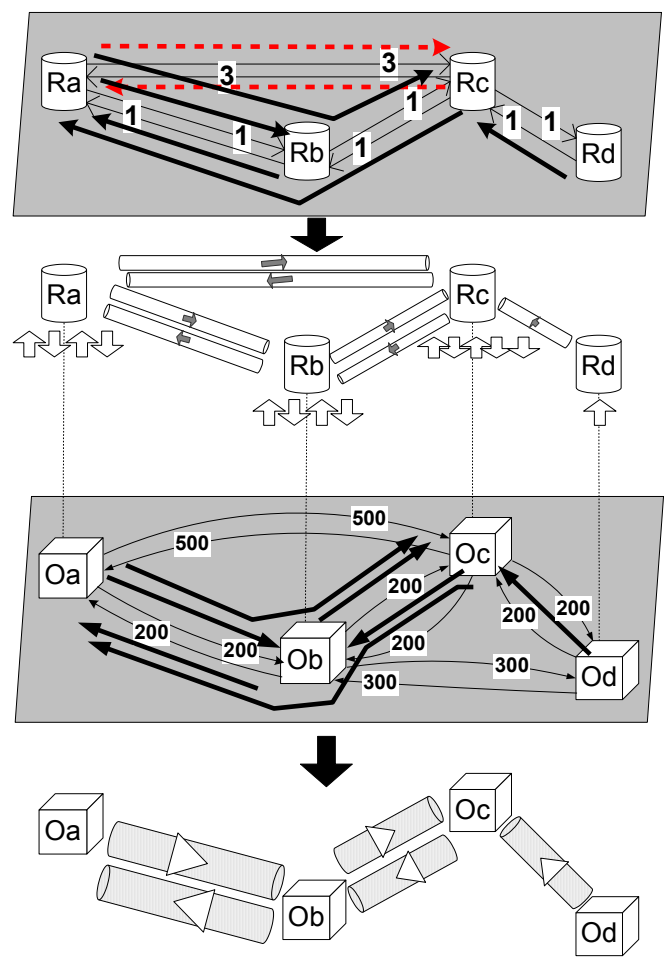

Fig 8 IP rerouting over static OTN: dimensioning including spare capacity.

\subsection{Shortest-path grooming (no IP recovery)}

In the shortest-path grooming scheme we only consider working IP flows and no survivability. Its dimensioning results can be used to determine an estimate of what would be needed extra to provide survivability (and providing a higher availability), assuming that you deploy shortest path routing in your IP network at all times. For grooming, there will be only a FFS and its dimensioning and this can be imagined as the same as in Fig. 8, except with the spare IP capacity flows (dotted lines) and its associated resources (light paths, ...).

\subsection{TE and bifurcated grooming (no IP recovery)}

For interface amount optimization, we were able to compare our single shortestpath routing schemes from above, with a scheme that also only considers working IP flows (grooming) but that allows split S-D flows over different paths (bifurcated routing) and unrestricted path routings for every split part. So, we can choose the number of paths over which the traffic demand between a source and a destination will be sent. Next, the individual routes for each path will be chosen, a decision which is part of traffic engineering (TE). We call this scheme "TE and bifurcated grooming". This means that a demand from a source to a destination can have several routes of which most of them will often be non-shortest paths. 
We looked at this scheme in order to quantify the performance loss due to the use of hop-count routing, a routing scheme in which the destination determines the shortest-path (destination based routing). TE and bifurcated grooming was obtained using a single layer grooming tool based on the single layer component of the heuristic method devised in [8]. As such, we obtained nearly optimal path routes and flows splitting. So, for the interface amount optimization, the results of $\mathrm{TE}$ and bifurcated grooming present a very good lower bound on the number of interfaces required in case the network operator wants a network that is supporting the working paths of a given traffic demand. We call it the "working traffic lower bound".

It presents the minimal amount of capacity that is needed to route the given traffic demand over a network graph. So, in TE and bifurcated grooming, we need to optimize: the logical topology, the capacity on the links, as well as the number of paths for each traffic S-D flow and the routing of each individual path. This is the most general scheme and its NP-hard optimization problem is also called multicommodity capacity installation (MCCI) (see [21]).

\subsection{Full demand mesh (FDM)}

In the Section 4.1 following, we will describe our logical topology design approaches. One such design is the Full demand mesh (FDM). It is mentioned here after the other schemes, because it can be applied for all the different single shortest-path routing schemes (survivable or non-survivable) and because it will be compared with them in the result sections. It cannot be applied to the TE and bifurcated grooming scheme.

\section{The logical topology designs}

In this section, we describe our approach for designing the logical topology. We first describe a basic design; next we describe our design optimisation method.

\subsection{Full demand mesh (FDM) with all weights equal to one}

This design installs a (directed) IP adjacency directly between every S-D router pair from the asymmetric traffic matrix that has a non-zero traffic demand. Consequently, there is no transit traffic in the IP routers. The nice property of this design is that there is no traffic to recover in case of a single router failure as there is not transit traffic in the IP router. All traffic originating or terminating in a 
failing router is lost. With respect to this property, the FDM design can be considered as equivalent to the other survivable schemes (IP rerouting over ASON and IP rerouting over static OTN) in terms of failure coverage. The topology can be depicted as a meshed graph obtained from the traffic demand matrix. In this sense, we call this design a "full demand mesh" (FDM). Note that it is different from a "full mesh" (FM) as the demand can have S-D router pairs that have no traffic request between them. This design serves as a reference topology to compare our optimized topology to. The FDM strategy uses as OSPF weights all ones (hopcount routing).

Feasible topologies for our survivable schemes need enough connectivity (see Section 2.3.1). It is important to describe how to cope with an FDM that is insufficiently connected - note that we did not encounter it in our case studies described below as the traffic demand of non-zero request was always sufficiently dense there. Our solution is to (1) add an amount of random edges to the insufficiently connected FDM, (2) evaluate its connectivity by doing the routing and the rerouting, and (3) finally repeat this procedure until we arrive at a mesh that has enough connectivity. Our solution could lead to a bad design, but this is where our basic approach (see next section) kicks in.

\subsection{Optimized logical topology}

In this section we first describe our basic optimization approach and next some improved variants of the optimization.

\subsubsection{Basic approach: local search edge deletion from the FDM as initial topology}

The basic approach starts from the FDM as a feasible initial design (see Section 4.1) and then applies a heuristic that only deletes edges from the initial design. The decision to delete edges comes from its effect on the objective value of the scheme under optimization. If the deletion of an edge leads to a better or equal objective, then the edge is removed forever. On the other hand, if the deletion of an edge leads to a worse objective then the edge is (remembered and) kept forever.

Another important decision in our algorithm was to keep an edge forever in the design if the load (a.k.a. filling) of at least one light path along that edge is higher than a certain fraction $\mathrm{f}$. The filling of such an edge is computed by doing the 
dimensioning of the failure free scenario (see Section 2.4). This process is called "fixing highly filled edges".

The weights of the design are the pre-determined weights of the initial design (ID). Recall that these weights remain the same for the whole optimization process. This implies that the shortest-path routing can only be influenced by removing edges. The pseudo code of the algorithm is given in Fig. 9. 
Notations:

- $I D=$ Initial logical topology design.

- $B D=$ Best logical topology design.

- $C D=$ Current logical topology design.

- MaxFilling $(e)=\frac{C_{-} \max }{C}$, where $C_{\text {is }}$ is the lightpath capacity and $C_{-}$max is the maximum traffic capacity routed over all the lightpaths installed along the edge e in the FFS.

Given:

- The context (implicitly passed to the algorithm)

- The fixed lightpath/wavelength/interface capacity $C$,

○ The physical topology structure including the weights on its links, and the fixed line-systems capacity $\boldsymbol{W}$,

- The current scheme under optimisation,

- The traffic demand matrix,

- For the context: the possibility to determine (1) whether the current topology design $C D$ under evaluation is feasible, and (2) whether it leads to a better objective value than the best feasible topology design $B D$ so far, and (3) the values MaxFilling(e) for the FFS.

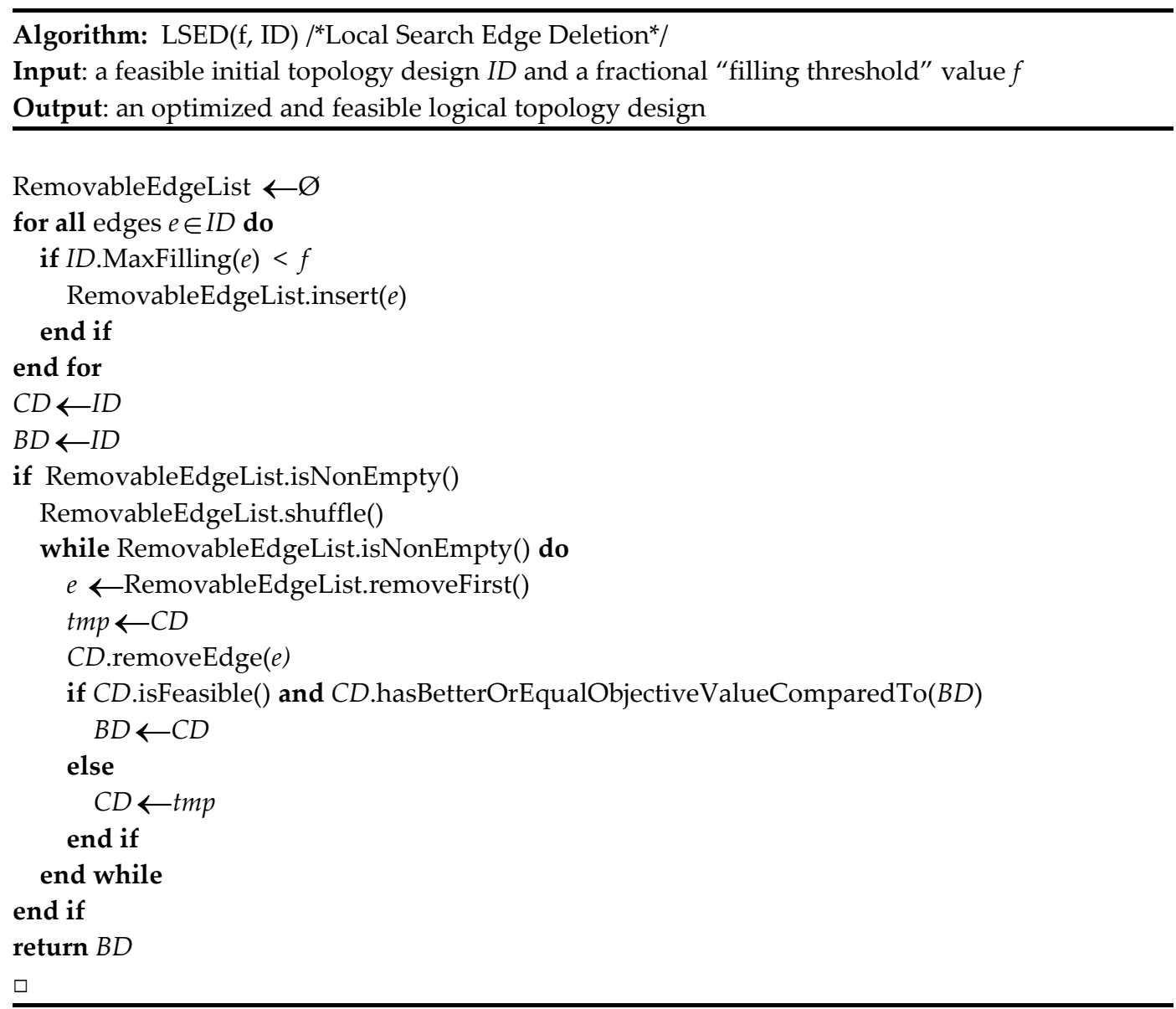

Fig 9 Pseudo code of the LSED algorithm.

\section{Discussion and naming}

We can say that this algorithm is a kind of local search using a steepest descent approach where neighbours are obtained by removing one edge from the current 
solution. We name it $\mathbf{L S E D}(<$ fillFraction>, <initial topology $>$ ) denoting that we use a local search approach that is deleting edges starting from an initial topology. In this section, we will focus on the FDM as initial topology: an example is $\operatorname{LSED}(0.8, \mathrm{FDM})$.

This approach is further enhanced by not considering an edge for removal once its removal has led to a worse solution (compared with the best solution so far). A further enhancement is "keeping highly filled edges forever".

An important decision was to shuffle the removable edges randomly. This serves as a kind of diversification. Alternatively, we also explored a non-random edge order by sorting them from lowly filled to highly filled (where the fillings are again calculated from the dimensioning of the FFS). We call this the "sorting variant". Surprisingly, we did not get better results for shortest-path grooming using it.

Another important decision was to keep the edge forever into the design, when the new objective value (after deletion of the edge) was worse than the best design so far but the new solution was still feasible. This opens the door for sub-optimal situations, but we assumed this would have only a slight effect on the quality of the outcome. Note that the other option for such a "worsening edge" would be "consider the edge later again". The decision to "keep such an edge for ever" was made to speed up the algorithm seriously. The decision of "fixing highly filled edges" was made for the same reasons.

The last important decision was to remove the edge forever from the design (instead of keeping it forever) when the new objective value was equal to the best known so far. We observed (when doing interface amount optimization) in general that this led to much better results for grooming and for IP rerouting over ASON, but made not much improvements for IP rerouting over static OTN.

We also observed that deleting some random edges from the initial FDM (in order to diversificate the optimization process) led in general to worse results, compared to the strategy that starts the optimization from the complete FDM.

\section{Conclusion}

The inspiring idea for this approach (using the FDM as initial design) was that the logical topology will be denser than the physical topology in an IP-over-optical network. Furthermore, the survivability for IP rerouting over ASON or static OTNs requires a more densely meshed logical topology than when only the 
grooming of working traffic is considered. This approach allows the detection of new feasible solutions when deleting edges starting from a feasible (enough connected) initial topology. It is a single approach that can be employed to different kinds of schemes and models to optimize. On the other hand, the optimization method does not employ much explicit "intelligence" from the model. All its intelligence is concentrated in the value of the objective and a bit in the fillings of the edges in the dimensioning of the FFS. Given the complexity of the models for the schemes, we see this as a computational efficient way of obtaining "intelligence" from the model. In our current implementations there is no reuse of computations regarding intelligence extraction from previously found solutions - except when using a previous design plus some perturbations, see below (Section 4.2.3). This is something that can be improved. Note that starting from a FDM (instead of starting for a FM) could exclude reaching optimal endsolutions, but is expected not to occur very often or to rarely lead to very bad solutions. In our case studies we almost always started from a FDM that was in fact an FM.

\subsubsection{Several "trials" of same algorithm}

We also investigated several trials of the basic algorithm in order to see the diversification effect of the random factors in the algorithm. These are the ordering of edges (IP links) that are to be deleted and the perturbations from the initial topology by adding random edges (see below). Of course, the optimality of the end-result is determined by the order of the deletion of the IP links. We denote $t$ trials of the LSED algorithm as $t \times \mathbf{L S E D}(. .$.$) : an example is 50 \times \operatorname{LSED}(0.7$, FDM).

\subsubsection{Alternative initial topologies}

The use of the FDM as initial design in LSED can further be improved by the following initial topology strategies.

\section{Initial topology design from a previous optimization}

We denote this initial design as OLT(<previous algorithm>), meaning that the optimized logical topology (design), that was the outcome of a previous application of our algorithm, is used as initial design. An important remark is that if we use several trials of an algorithm with an OLT as initial topology then for 
every trial the same initial topology is used: so $10 \times \operatorname{LSED}(f 1$, OLT(LSED(f2, FDM $)$ )) essentially means that $10 \times \operatorname{LSED}(f 1$, ID) is carried out, working every time on the single ID equal to an outcome of $\operatorname{OLT}(\operatorname{LSED}(f 2, \mathrm{FDM}))$.

We observed that first optimizing with a lower filling value (e.g., $f=0.7$ ) for fixing highly filled edges and then re-optimizing - with the first outcome as the initial topology - using a higher filling value (e.g., $f=1.0$, meaning no fixing at all) often lead to improvements on the first outcome of the order of $5 \%$ to $10 \%$.

\section{Initial topology design from a given design PLUS some random edges}

We denote this initial topology: PERFDM(<percentage $>,<$ given design>). PERFDM stands for "Percentage Extra Random links from the FDM". It means that it adds an extra percentage of IP links (edges) to the given design. The added IP links are chosen randomly from the adjacencies that are not yet present in the given (previous) design, but are existent in the FDM - assuming that the given design will be a subset of the FDM. The same weights are used as in the previous given design for its edges and the weights from the FDM for the newly added random edges (where the weights from the previous design also came from the FDM originally). Adding of random edges forms a kind of diversification. For example PERFDM(10\%, OLT(LSED(0.5, FDM)), will return a randomly different initial topology every time it is asked for, by adding different random edges to the given design. That single given design will be an outcome of $\operatorname{LSED}(0.5, \mathrm{FDM})$, and is thus denoted as $\operatorname{OLT}(\operatorname{LSED}(0.5, \mathrm{FDM}))$.

\section{Best example}

An example of a complete algorithm using PERFDM is $10 \times \operatorname{LSED}(0.8$, $\operatorname{PERFDM}(10 \%, \operatorname{OLT}(\operatorname{LSED}(0.5, \mathrm{FDM}))))$. Ten trials of $\operatorname{LSED}(0.8,<$ initial topology>) are applied to the initial topology. There are ten different initial topologies. Each time it is formed by adding $10 \%$ random edges to a single given design, being $\operatorname{OLT}(\operatorname{LSED}(0.5, \mathrm{FDM}))$. This is our most complicated algorithmic approach. With unspecified parameters it is denoted as $\boldsymbol{t} \times \mathbf{L S E D}(\boldsymbol{f} \mathbf{1}$, $\operatorname{PERFDM}(p, \operatorname{OLT}(\operatorname{LSED}(\mathbf{f}, \operatorname{FDM})))))$. These algorithm instances allowed us to find our best solutions. 


\section{Case studies and results}

In this section we present the results obtained from two different case studies that we call "Euro12" and "Lion14". We first describe the scenario and the results of the two case studies separately and afterwards compare them. For each scenario we will present the results obtained for the two considered optimisation objectives (see Section 2.5.3).

\section{1 “Euro12" case study}

The physical topology of the Euro12 case is a European optical transport network that consists of 12 nodes and 17 bidirectional links, which we have converted into 34 unidirectional links. The resultant average nodal degree is 2.83 . It is a downsized version derived from a network with more nodes that was used as a pan-European reference network topology. It is obtainable from [13].

The traffic demand is modelled by a symmetric matrix with a total demand of $630.6 \mathrm{Gbps}$. It is a downsized version of the traffic matrix in [13], which estimates pan-European traffic for the year 2002. The estimation is based on a data (modem and IP) and voice traffic model that relies on the population, number of (nonproduction) employees and number of internet hosts for each of the city regions. As shown in Fig. 10, the traffic matrix used in our simulations gives rise to a fairly uniform traffic pattern, with an average of about $53 \mathrm{Gbps}$ of ingoing/outgoing traffic at each node and an average of $4.78 \mathrm{Gbps}$ of traffic between each node pair. Since in this case study the value assigned to the constant $\mathrm{C}$ is $10 \mathrm{Gbps}$, a connection (the total flow) between a source and a destination node needs on average the $48 \%$ of $\mathrm{C}$. 


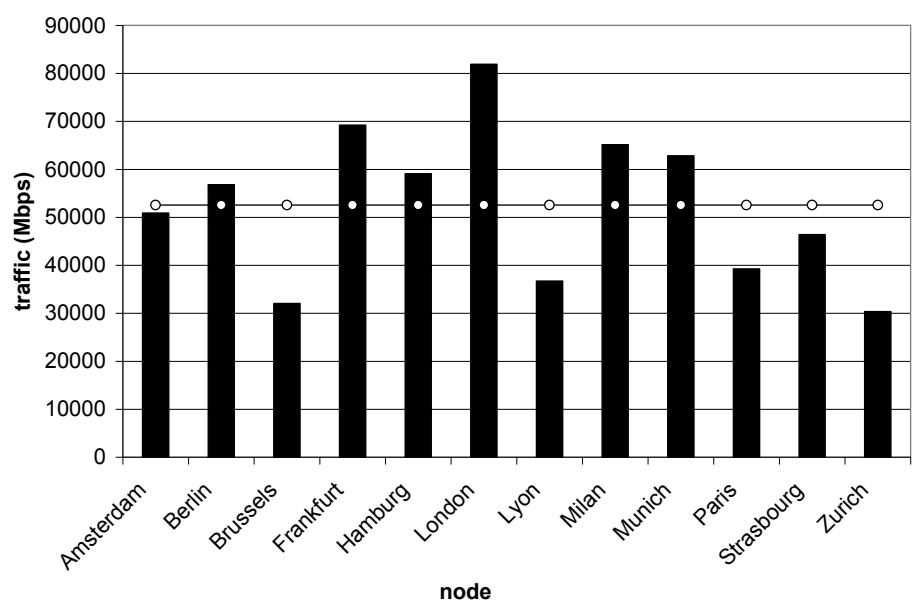

Fig 10 Ingoing/outgoing traffic (black bars) at each node in the Euro12 case study, together with the average over all nodes (white dots)

\subsubsection{Interface amount optimization}

The results are summarized in Table 1 and they are the best ones obtained by using one of the optimization approaches described in Section 4.2. We have also calculated the lower bound for the number of needed interfaces with this formula: $2 \times($ total traffic amount / C). We call this value the "interface amount lower bound".

Let us have first a look at the comparison between the non-survivable shortestpath grooming scheme, whose logical topology optimization under the Euro12 scenario requires 208 IP router interfaces, and the three different survivable schemes, namely IP rerouting over ASON, IP rerouting over static OTN and FDM. Recall that in all of them we have assumed to use shortest-path routing as well.

The additional cost for providing survivability is $2 \%$ ( 212 interfaces needed) for IP rerouting over ASON, 14\% (236 interfaces needed) for IP rerouting over static OTN and 27\% (264 interfaces needed) for FDM design. Reminding that FDM is the simplest scheme to implement (no logical topology algorithm design required) and the best one in terms of QoS (it is survivable and no restoration takes place since there is no transit traffic in the IP routers), we can conclude that an additional cost of only $27 \%$ is a surprising result. The key result is the comparison between ASON and static OTN which we have often mentioned along this paper. The resources reuse enabled by the dynamic reconfigurability of an ASON, allows us to save about the $12 \%$ (24 interfaces) of the cost, with respect to a static OTN, to route and reroute IP traffic. 
Table 1 also reports the cost required by the TE and bifurcated grooming scheme (the working traffic lower bound, see Section 3.3). Using this value as a reference to compare the performance loss when using shortest path routing (which does not allow flow splitting), we can conclude that the cost penalty is about 25\% (42 extra interfaces needed).

The benefit of the flow splitting is emphasized by two extra experiments we run. In the first one, we used the logical topology design optimized for TE and bifurcated grooming scheme and applied shortest-hop routing on it. In the second one, we used the shortest path routing scheme with the IP link weights equal to the length (in kilometers) of the corresponding path in the optical layer. As expected, both experiments led to results worse than our optimized shortest-path grooming design.

Table 1 Results of the Euro12 case for interface amount optimization.

\begin{tabular}{|l|l|r|}
\hline scheme(s) & design & $\begin{array}{l}\text { number } \\
\text { of } \\
\text { interfaces }\end{array}$ \\
\hline $\begin{array}{l}\text { shortest-path } \\
\text { grooming }\end{array}$ & optimized using one of the approaches in 4.2 & 208 \\
\hline $\begin{array}{l}\text { TE and bifurcated } \\
\text { grooming }\end{array}$ & optimized using a single layer grooming tool & 212 \\
\hline $\begin{array}{l}\text { IP rerouting over } \\
\text { ASON }\end{array}$ & optimized using one of the approaches in 4.2 \\
\hline $\begin{array}{l}\text { IP rerouting over } \\
\text { static OTN }\end{array}$ & optimized using one of the approaches in 4.2 & 236 \\
\hline $\begin{array}{l}\text { shortest-path } \\
\text { grooming, IP } \\
\text { rerouting over ASON } \\
\text { and IP rerouting over } \\
\text { static OTN }\end{array}$ & FDM & 264 \\
\hline $\begin{array}{l}\text { interface amount } \\
\text { lower bound }\end{array}$ & $/$ & 127 \\
\hline
\end{tabular}

\subsubsection{Complete IP plus optical layer cost optimization}

The best results, together with the optimization approach used to obtain them, are presented in Table 2, where the total cost refers, in this case, to the total number of IP interfaces needed plus the costs assigned to every resource component of the line-systems needed in the optical layer. The capacity of a line-system is $\mathrm{W}=40$ wavelengths and for a more detailed description of the cost number the reader is referred to [10]. 
As done for the interface amount optimization, we focus on the comparison between the non-survivable shortest-path grooming (31 line systems installed and a total cost of 2150) and the already mentioned survivable ones, so that we can estimate the cost difference between the IP rerouting over ASON and the IP rerouting over static OTN, evaluated to $10 \%$. In fact, the former scheme requires an additional cost equal to $4 \%$ (33 line systems installed and a total cost of 2239) in order to provide survivability and the latter one requires the 14\% (33 line systems installed and a total cost of 2454) of extra equipment. Concerning the use of the FDM scheme in designing the logical topology, the results obtained (26\% of additional cost) are in line with the ones presented in Table 1, meaning that it is still an attractive value with respect to its simplicity and the QoS offered.

The fact that the results obtained for the two different objectives do not provide notable differences is emphasized by our observation of the IP interfaces number for the interface amount and the complete IP plus optical layer cost optimization. It remains in the same range, suggesting that the interface amount optimization is an interesting first-approach-subproblem.

Table 2 Results for the Euro12 case and the complete IP plus optical layer cost objective.

\begin{tabular}{|c|c|c|}
\hline scheme(s) & design & $\begin{array}{l}\text { total } \\
\text { cost }\end{array}$ \\
\hline $\begin{array}{l}\text { shortest-path } \\
\text { grooming }\end{array}$ & $50 \times \operatorname{LSED}(1.0, \operatorname{PERFDM}(20 \%, \mathrm{OLT}(\operatorname{LSED}(0.8, \mathrm{FDM}))))$ & 2150 \\
\hline $\begin{array}{l}\text { IP rerouting } \\
\text { over } A S O N\end{array}$ & $50 \times \operatorname{LSED}(1.0$, PERFDM(10\%, OLT(LSED $(0.8$, FDM $))))$ & 2239 \\
\hline $\begin{array}{l}\text { IP rerouting } \\
\text { over static OTN }\end{array}$ & $50 \times \operatorname{LSED}(1.0, \operatorname{PERFDM}(10 \%, \mathrm{OLT}(\operatorname{LSED}(0.5, \mathrm{FDM}))))$ & 2454 \\
\hline $\begin{array}{l}\text { shortest-path } \\
\text { grooming, IP } \\
\text { rerouting over } \\
\text { ASON and IP } \\
\text { rerouting over } \\
\text { static OTN }\end{array}$ & FDM & 2700 \\
\hline
\end{tabular}

\section{Approach suggestions}

As shown in Table 2, the cost evaluation in case of complete IP plus optical layer is made using different parameters in the logical topology design optimization of each routing and rerouting scheme. This is due to the fact that no specific parameter setting appeared to be the most suited for all the schemes. For this reason, we provide some guidelines derived from observing the Euro12 case and concerning the parameters in the general algorithms $t \times \operatorname{LSED}(f 1, \operatorname{OLT}(\operatorname{LSED}(f 2$, 
$\operatorname{FDM})))$ and $\operatorname{LSED}(f 1, \operatorname{PERFDM}(p, \operatorname{OLT}(\operatorname{LSED}(f 2, \operatorname{FDM})))))$. The improvements when using PERFDM to add random edges to a previous design can be $6 \%$ for shortest-path grooming and 3\% for IP rerouting over ASON, but almost nothing for IP rerouting over static OTN. In general, when using the former one, we suggest the following parameters: $0.5<f 2<0.8, f 1=1, t=10$. Sometimes, we observed better results using the more intensive latter one with $0.5<f 2<0.8$, with $f 1=1$, with $p$ around $10 \%$ or $20 \%$ and $t$ being higher, e.g., 50 . Note that we always choose $f 1$ equal to 1.0, as it tries to delete every adjacency (instead of just keeping the highly filled ones a priori, see Section 4.2.1) and often leads to better results. Concerning the parameters $f 2$ and $p$, while we did not observe any specific optimal setting for the IP rerouting over ASON scheme, we suggest $p=10 \%$ and $f 2=0.5$ for the IP rerouting over static OTN scheme and $p=20 \%$ and $0.5<f 2<$ 0.8 for the shortest-path grooming scheme. Concerning Euro12, very high values of the number of trials (e.g., $t$ equal to 1000 instead of 50) could lead to better results at the expense of a proportional increase in time consumption. This was especially true for shortest-path grooming (8\%). The improvements are less for IP rerouting over ASON (5\%) and for IP rerouting over static OTN (3\%).

\section{Other results}

We have also changed the value $\mathrm{C}$ and scaled the original traffic matrix of Euro12.

In the former case, a value $\mathrm{C}$ equal to $2.5 \mathrm{Gbps}$ leads to a high cost increase $(8.6 \%)$ with respect to the base case with $\mathrm{C}$ equal to $10 \mathrm{Gbps}$. Obviously, this is due to the relatively high amount of traffic streams in Euro12. In fact, with this new value for $\mathrm{C}$ equal to $2.5 \mathrm{Gbps}$ the average amount of traffic between a node pair is about $191 \%$ of C.

In general, a capacity increase by a factor four leads to only a cost increase by a factor three. Thus, the inverse economy of scale by going from 10 Gbps to 2.5 Gbps interfaces should lead to an increase of $33 \%$ or more in total cost. This is not achieved due to the granularities in the model.

In the latter case, our experiments with a scaled $(\times 2, \times 4, \times 8, \times 16)$ version of the traffic matrix (keeping $\mathrm{C}$ equal to $10 \mathrm{Gbps}$ ) presented relatively huge traffic flows that drive the design for all the routing schemes close to the FDM. In this way, we cannot appreciate the benefits of an ASON over a static OTN in routing and 
rerouting traffic. In fact, there are only a few percents of difference in case of doubled traffic and almost no difference for the other higher scaled matrices.

\section{Effect of optimizing logical topology design for additional survivability}

To further focus on the effect that our optimization strategy has on the network cost, we ran some experiments starting from a topology optimized for working traffic alone (the grooming scheme) and, without running our design optimisation algorithm, we only added capacity to reach a survivable network solution. We observed that a network operator would be charged an additional cost of $3 \%$ for IP rerouting over ASON scheme and 15\% for IP rerouting over static OTN. This difference illustrates the importance of the LTD optimisation with a specific target for the scheme and its objective. IP rerouting over static OTN performs much worse than IP rerouting over ASON if it is adopted on a logical design that is not specific drafted for it - namely on the design for grooming that turned out to be sufficiently connected. The results are reported in Table 3 and it can be considered as an extra advantage of IP rerouting over ASON in providing survivability. In fact, it makes the transition from a non-survivable groomed solution to a survivable one less painful compared to a static OTN.

Table 3 Effect of optimizing when providing survivability

\begin{tabular}{|l|r|rr|}
\hline scheme(s) & $\begin{array}{l}\text { total cost (optimized } \\
\text { design) }\end{array}$ & \multicolumn{2}{|l|}{$\begin{array}{l}\text { total cost (shortest-path grooming } \\
\text { design) }\end{array}$} \\
\hline $\begin{array}{l}\text { shortest-path } \\
\text { grooming }\end{array}$ & 2150 & same design: 2150 \\
\hline $\begin{array}{l}\text { IP rerouting over } \\
\text { ASON }\end{array}$ & 2239 & 2308 \\
\hline $\begin{array}{l}\text { IP rerouting over } \\
\text { static OTN }\end{array}$ & 2454 & & 2818 \\
\hline
\end{tabular}

\section{2 “Lion14" case study}

The physical topology is of the Lion14 case is based on a national IP-over-optical network in Italy. The physical topology consists out of 14 nodes and of 28 bidirectional links, which we have converted to 56 unidirectional links. The resulting average nodal degree is 4 .

The IP traffic modeled represents the traffic of various services (data from 2001) like IP telephony, e-mail, web-based services (web servers and internet uplinks) and video on demand. Fig. 11 and Fig. 12 represent the client/server traffic 
demand and show that large server farms are located in two cities, while two other cities have smaller servers. In other words, there are four cities producing most of the server traffic and while client traffic is a bit more balanced over the cities, it is still concentrated in the four most important ones. The result is a highly asymmetric traffic matrix, with a total demand of $127.1 \mathrm{Gbps}$, an average of 9.1 Gbps of ingoing/outgoing traffic at each node and an average of 0.7 Gbps of traffic between each nodes pair. More details on this network and its traffic assumptions can be found in [14].

The value used for the router port capacity and the light path capacity (C) is 2.5 Gbps. Although this value is smaller than the Euro12 case study, we can consider Lion14 as a low traffic case, since a connection between a source and a destination node only needs the $28 \%$ of C.

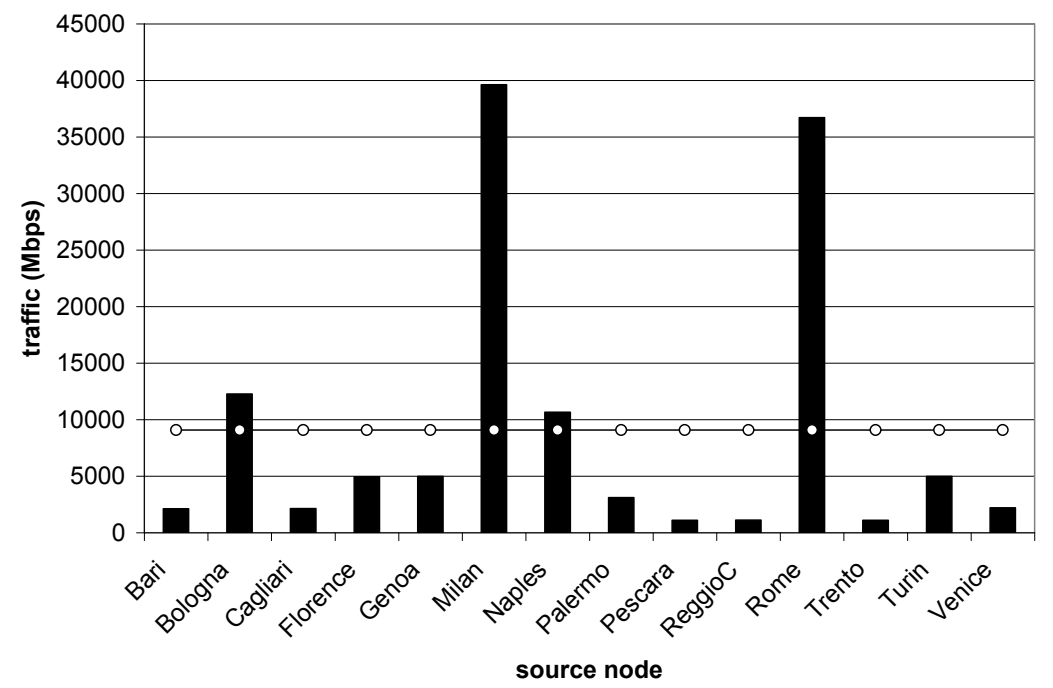

Fig. 11 Outgoing traffic (black bars) at each node in the Lion14 case study, together with the average over all nodes (white dots). 


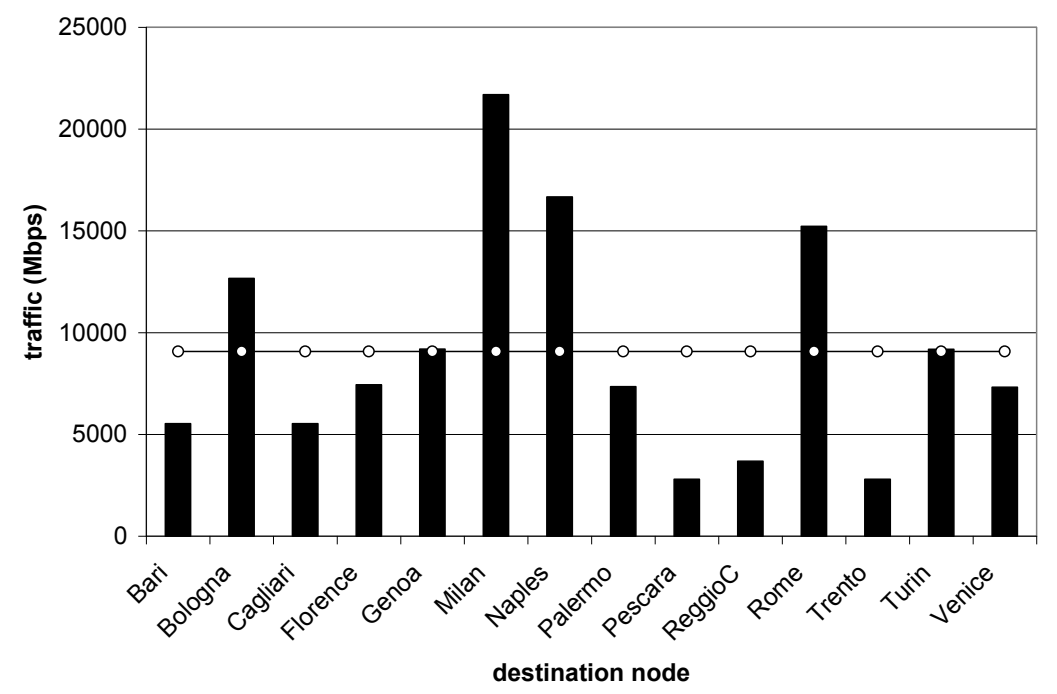

Fig 12 Ingoing traffic (black bars) at each node in the Lion14 traffic demand, together with the average over all nodes (white dots).

\subsubsection{Interface amount optimization}

The results are summarized in Table 4 and, as done in the Eurol 12 case, they are the best ones obtained by using one of the optimization approaches described in Section 4.2. The additional cost for providing survivability, with respect to the shortest-path grooming scheme where only working traffic is considered (172 interfaces needed), is evaluated to $8 \%$ for IP rerouting over ASON (186 interfaces needed), 16\% for IP rerouting over static OTN (200 interfaces needed) and 133\% (400 interfaces needed) for the FDM design. The cost difference between ASON and static OTN in routing and rerouting IP traffic is $8 \%$ and the performance loss due to the use of shortest-path routing in our schemes is evaluated to $18 \%$ of additional cost compared to the TE and bifurcated grooming.

Table 4 Results on the Lion14 case for interface amount optimization.

\begin{tabular}{|l|l|r|}
\hline scheme(s) & design & $\begin{array}{l}\text { number of } \\
\text { interfaces }\end{array}$ \\
\hline shortest-path grooming & optimized using one of the approaches in 4.2 & 172 \\
\hline $\begin{array}{l}\text { TE and bifurcated } \\
\text { grooming }\end{array}$ & optimized using single layer grooming tool & 146 \\
\hline IP rerouting over ASON & optimized using one of the approaches in 4.2 & 200 \\
\hline $\begin{array}{l}\text { IP rerouting over static } \\
\text { OTN }\end{array}$ & optimized using one of the approaches in 4.2 & 400 \\
\hline $\begin{array}{l}\text { shortest-path grooming, } \\
\text { IP rerouting over ASON } \\
\text { and IP rerouting over } \\
\text { static OTN }\end{array}$ & FDM & 102 \\
\hline $\begin{array}{l}\text { interface amount lower } \\
\text { bound }\end{array}$ & $/$ & \\
\hline
\end{tabular}




\subsubsection{Complete IP plus optical layer cost optimization}

The results are presented in Table 5. The additional total cost, compared with shortest-path grooming (25 line-systems installed and a total cost of 1013), is 28\% (34 line-systems installed and a total cost of 1294) for IP rerouting over ASON scheme, 35\% (35 line-systems installed and a total cost of 1363) for IP rerouting over static OTN and 124\% (total cost of 2268) for the FDM design. When considering also the optical layer in the total cost, the difference between IP rerouting over ASON and IP rerouting over static OTN is evaluated to 7\%.

Table 5 Results for the Lion14 case and the complete IP plus optical layer cost objective.

\begin{tabular}{|c|c|c|}
\hline scheme(s) & design & $\begin{array}{l}\text { total } \\
\text { cost }\end{array}$ \\
\hline $\begin{array}{l}\text { shortest-path } \\
\text { grooming }\end{array}$ & $\begin{array}{l}10 \times \operatorname{LSED}(1.0, \text { PERFDM }(10 \% \text {, OLT }(\operatorname{LSED}(0.8 \text {, } \\
\text { FDM }))))\end{array}$ & 1013 \\
\hline $\begin{array}{l}\text { IP rerouting over } \\
\text { ASON }\end{array}$ & $\begin{array}{l}10 \times \operatorname{LSED}(1.0, \operatorname{PERFDM}(10 \%, \mathrm{OLT}(\operatorname{LSED}(0.8, \\
\text { FDM }))))\end{array}$ & 1294 \\
\hline $\begin{array}{l}\text { IP rerouting over } \\
\text { static OTN }\end{array}$ & $\begin{array}{l}50 \times \operatorname{LSED}(1.0, \text { PERFDM }(10 \%, \text { OLT }(\operatorname{LSED}(0.8 \text {, } \\
\text { FDM }))))\end{array}$ & 1363 \\
\hline $\begin{array}{l}\text { shortest-path } \\
\text { grooming, IP } \\
\text { rerouting over } \\
\text { ASON and IP } \\
\text { rerouting over } \\
\text { static OTN }\end{array}$ & FDM & 2268 \\
\hline $\begin{array}{l}\text { IP rerouting over } \\
\text { ASON }\end{array}$ & result from [3] & 1766 \\
\hline
\end{tabular}

We also added to Table 5 the total cost of the IP rerouting over ASON obtained in [3]. In that paper, the design target is a low cost failure free operation. The design used shortest-path weights in the IP layer based on the estimation of the linear cost of a light path in the optical layer. Although in the optical layer the cost of the protected light path was taken into account, the results indicate that specific topology optimization for IP rerouting and for the objective in question, leads to important cost gains of $36 \%$ !

Also in the Lion 14 case study, we ran some experiments with a scaled traffic demand. The results indicate that the benefits of an ASON over a static OTN remain around a $5 \%$ concerning the total cost when rerouting IP traffic. 


\section{3 “Euro12" versus "Lion14"}

In this paragraph we will compare the main results obtained for the two case studies. Before differentiating the results obtained for the two objectives, it is worthwhile to underline that in the Euro12 case study the total traffic demand is five times larger than the one in the Lion14 case, while using a four times bigger value for the IP router interface and light path capacity - combined this leads to a scaling of about 5/4). In addition, there is a notable difference in the traffic pattern of the two cases. While the Euro12 scenario has a symmetric quite uniform traffic demand matrix, the Lion14 one presents four important traffic nodes (client + server) that are giving rise to a highly asymmetric traffic demand.

- Interface amount optimization. In the Lion 14 case study, all the survivable schemes have a worse performance compared to the survivable shortest-path grooming scheme. Nevertheless, while IP rerouting over ASON and over static OTN, costs remain in the same range (a difference of $6 \%$ for the former and $2 \%$ for the latter). The FDM design undergoes a cost increase of $106 \%$. These results indicate that the logical topology optimization presented in this work not only provides a good performance, but is even necessary in case of non-uniform traffic demands. Another remark is about the performance loss when using shortest path compared to the flow-splitting technique of the TE and bifurcated grooming scheme (the working path lower bound). In the Lion14 case, the shortest path routing has an additional cost slightly lower than the one in the Euro12 case. This is partly due to the large number of small IP flows in Lion14. In fact, in the Euro1 2 case, more traffic flows are directly sent from source to destination in the logical topology, as they are quite large compared to the capacity $\mathrm{C}$. The comparison between the two case studies is summarized Table 6.

Table 6 Comparison between the two case studies for the IP interface amount optimization objective.

\begin{tabular}{|l|r|r|}
\hline scheme or design or routing option & \multicolumn{1}{|l|}{ Euro12 } & \multicolumn{1}{|l|}{ Lion 14} \\
\hline IP rerouting over ASON & $2 \%$ & $8 \%$ \\
\hline IP rerouting over static OTN & $14 \%$ & $16 \%$ \\
\hline ASON versus static OTN & $12 \%$ & $8 \%$ \\
\hline \multicolumn{3}{|l|}{} \\
\hline FDM & $27 \%$ & $133 \%$ \\
\hline shortest-path penalty & $25 \%$ & $18 \%$ \\
\hline
\end{tabular}




\section{- Comparison for the complete IP plus optical layer cost optimization.}

The average nodal degree in the physical topology is 2.83 in the Euro12 case and 4 in the Lion 14 one. Concerning the IP topology, this value is about 7 in Euro12 and 3 in Lion14, indicating that our optimization process chooses for a sparser logical design in Lion14 in order to groom more extensively IP flows into a light path, while installing more direct logical links in the Euro12 case that has larger S-D flows. At the same time, the higher amount of the logical links in the Euro12 case seems not to be penalized in the optical layer, because the physical topology of the Euro12 case is sparser and drives the grooming of wavelengths into linesystems via the shortest-path routing. While scaling up the traffic of the Lion14 demand matrix, the better grooming of IP flows into light paths remained. This justifies the higher cost (around $20 \%$ of difference) in the Lion14 for the survivable schemes (IP rerouting over ASON and IP rerouting over static OTN). In general: efficient grooming, with respect to the failure free scenario alone, leads to more transit traffic that subsequently demands high additional backup bandwidth installation. Thus, the topology optimisation needs to balance these two contributions to the total cost. Concerning the FDM design, supposing complete IP plus optical layer cost optimisation, the relative cost is much higher for the Lion14 case study. This illustrates the importance of optimizing the logical topology. The comparison between the two case studies concerning the complete IP plus optical layer cost optimization is summarized in Table 7.

Table 7 Comparison between the two case studies for the complete IP plus optical layer cost optimization.

\begin{tabular}{|l|r|r|}
\hline scheme or design & \multicolumn{1}{|l|}{ Euro12 } & \multicolumn{1}{l|}{ Lion 14} \\
\hline IP rerouting over ASON & $4 \%$ & $28 \%$ \\
\hline IP rerouting over static OTN & $14 \%$ & $35 \%$ \\
\hline ASON versus static OTN & $12 \%$ & $7 \%$ \\
\hline & & \\
\hline FDM & $26 \%$ & $124 \%$ \\
\hline
\end{tabular}

\subsection{Other study}

We also studied a traffic demand among seven nodes with a connection for every node pair that was a random integer between 0 and $2500 \mathrm{Mbps}$. Scaled versions of this demand revealed that when the average size of S-D traffic demand 
is about $50 \%$ or more of the capacity of a light path/interface/wavelength, the FDM turns out to be a good design option for interface amount optimization while being simple for deployment. For complete IP plus optical layer cost optimization, we used several random bi-connected physical topologies of various degrees (with all weights equal to one, because distance information was not available). These different physical topologies altered the shortest-path routing in the optical layer and we were able to improve the multi-layer designs for a given scheme by $10 \%-15 \%$. A sparser physical topology seems to be advantageous, because it permits more grooming of light paths into line-systems, when we are using shortest-path routing.

\section{Conclusions}

This paper demonstrates the use of an ASON for IP router failure recovery. The advantage of an ASON in comparison to a static OTN is that a better reuse of resources is possible. This is due to the dynamic reconfigurability of the logical topology, allowing the setting up and tearing down of light paths at the time of the failure event. This flexibility enables extra reuse of router interface cards (IP layer) and wavelengths of affected working paths (optical layer). It is supplementary to the well-known sharing of backup bandwidth in case of nonshared risks that comes from the packet-oriented nature of the IP layer. We developed a tool to quantify its cost benefit for different physical and logical topology designs and different traffic demand matrices. The tool takes into account the total IP-over-optical layer cost including transponders, WDM multiplexers, IP router cards, optical interfaces, amplifiers, etc. We assumed single node failures (leading to a loss of all traffic terminated traffic by the node), shortest-hop routing and no fibre digging costs.

An algorithm to optimize the logical topology in case of different schemes was developed. The non-survivable shortest-path grooming scheme was used as a reference strategy, but the main focus was to compare the two survivable schemes, IP rerouting over static OTN and over ASON, against each other. Most important, it was demonstrated that logical topology optimization specific for the model and objective in question can make prominent savings, even when hopcount routing is used. Our results also indicated that the advantage of ASONs over static OTNs for IP rerouting is roughly in the range of 5\% to $15 \%$ in total 
cost. This is less than expected and is due to the specific topology optimisation. When IP rerouting over ASON and IP rerouting over static OTN are used to add survivability to the same topology design (that fits well the working traffic alone), the latter could easily perform $25 \%$ worse. The exact savings number depends on the traffic pattern, its intensity and the physical topology (and cost data). It was showed that the drawback of shortest-hop grooming can be large compared with TE and bifurcated grooming (about $20 \%$ to $25 \%$ ). The very simple logical topology design of a full demand mesh (FDM) - that can be attractive from an operator's deployment perspective - performs quite well, compared with an optimized solution, especially when the Source-Destination (S-D) IP traffic flows are large enough (i.e., when they are on average $50 \%$ or more of the capacity of the light paths) and when their average traffic intensity is not dominated by outliers (large traffic demands from important server or clients nodes). In general, one can say that it is a good choice for all schemes to install a direct link between an S-D node pair if its S-D traffic flow is more than $50 \%$ of the capacity of the light paths. We observed several times that despite its survivability capabilities, the IP rerouting over ASON scheme is more related to the non-survivable shortest-path grooming scheme than to the IP rerouting over static OTN scheme. For example, the degree of an optimized logical topology for the case of IP rerouting over static OTN is often higher than in case of IP rerouting over ASON. The value of the latter's nodal degree is closer to that of the grooming scheme. IP rerouting over ASON can also make an easy upgrade from a previously optimized and deployed design for shortest-path grooming. Only 7\% extra expenditures are needed in that case. It was demonstrated that efficient grooming (with respect to the failure free scenario alone) lead to more transit traffic than our two survivable schemes. The survivable schemes are favoring direct (end-to-end) logical IP links so that the additional backup bandwidth installation for when a transit router fails can be avoided. Although not being the focus of this paper, we have indications that it is beneficial to also optimize the physical topology when using shortestpath routing for the light paths and that this can make an extra saving of another $10 \%$. 


\section{Acknowledgements}

Adelbert Groebbens is Research Assistant with the Flemish Fund for Scientific Research (F.W.O.-Vlaanderen). Didier Colle would also like to thank the IWT for its financial support for its postdoctoral grant. The work was partly funded by the Flemish Government through the project(s) FWO G.0315.04, IWT-GBOU ONNA and IWT-ITEA TBONES.

\section{References}

[1] D. Colle, S. De Maesschalck, A. Groebbens, B. Puype, Q.Yan, I. Lievens, M. Pickavet, P. Demeester, Cost-efficient deployment of survivable next-generation IP-over-optical networks, (invited) proc. of SPIE, Optical Transmission Systems and Equipment for WDM Networking II, 5247, Orlando, Florida (September 2003), 215-229, doi: 10.1117/12.512371

[2] J.F. Labourdette, E. Bouillet,S. Chaudhuri, Role of Optical Network and Spare Router Strategy in Resilient IP Backbone Architecture, in proc. $4^{\text {th }}$ International Workshop on the Design of Reliable Communication Networks (DRCN) 2003, Banff, Alberta, Canada ( October 2003), 244-253, doi: 10.1109/DRCN.2003.1275363

[3] S. De Maesschalck, D. Colle, A. Groebbens, C. Develder, I. Lievens, P. Lagasse, M. Pickavet, P. Demeester, F. Saluta, M. Quagliotti, Intelligent optical networking for multilayer survivability, IEEE Communications Magazine, 40(1), 42-49 (January 2002), doi: $10.1109 / 35.978048$

[4] C. Chigan, G. Atkinson, R. Nagarajan, T.G. Robertazzi, Mathematical Programming for Joint Restoration of IP over Optical Networks, in proc. Conference on Information Sciences and Systems, Princeton University, Princeton N.J (March 2002)

[5] L. Lei, A. Liu, Y. Ji , A joint resilience scheme with interlayer backup resource sharing in IP over WDM networks, IEEE Communications Magazine, 42(1), 78-84 (January 2004), doi: 10.1109/MCOM.2004.1262165

[6] A.L. Chiu, J. Strand, An agile optical layer restoration method for router failures, IEEE Network, 17(2), 38-42 (March/April 2003), doi: 10.1109/MNET.2003.1188285

[7] V. R. Konda, T. Chow, Algorithm for traffic grooming in optical networks to minimize the number of transceivers, in proc. 2001 IEEE Workshop on High Performance Switching and Routing (HPSR 2001), Dallas, Texas (May 2001), 218-221, doi: 10.1109/HPSR.2001.923635

[8] S. De Maesschalck, M. Pickavet, D. Colle, S. Verbrugge, Q. Yan, B. Puype, P. Demeester, Efficient Multi-layer Traffic Grooming in an IP/MPLS-over-Optical Network, European Transactions on Telecommunications, 16(4), 329-347 (July/August 2005), doi: 10.1002/ett.1020 
[9] H. Zhu, H. Zang, K. Zhu, and Biswanath Mukherjee, A Novel, Generic Graph Model for Traffic Grooming in Heterogeneous WDM Mesh Networks, IEEE/ACM Transaction on Networking, 11(2), 285-299 (April 2003), doi: 10.1109/TNET.2003.810310

[10] J. Derskacz, M. Leszczuk, K. Wajda, R. Leone, G. Monari, I. Lievens, S. De Maesschalck, S. Verbrugge, D. Colle, IP/OTN Cost Model and Photonic Equipment Cost Forecast - IST project LION, in proc. 4th Workshop on Telecommunications Techno-Economics, Rennes, France (May 2002)

[11] A. Groebbens, D. Colle, S. De Maesschalck, M. Pickavet, P. Demeester, Logical topology optimization for dynamic multi-layer recovery schemes, in proc. $4^{\text {th }}$ International Workshop on the Design of Reliable Communication Networks (DRCN) 2003, Banff, Alberta, Canada (October 2003), 47-52, doi: 10.1109/DRCN.2003.1275337

[12] A. Groebbens, D. Colle, M. Pickavet, P. Demeester, Logical Topology Design Minimizing the Cost of Survivable IP/MPLS-over-OTN Architectures, in proc. $7^{\text {th }}$ INFORMS Telecommunications Conference, Boca Raton, Florida (March 2004), 239-241

[13] LION/COST266 reference networks, http://www.ibcn.intec.ugent.be/INTERNAL/NRS/index.html

[14] D. Colle, S. De Maesschalck, I. Lievens, M. Pickavet, P. Demeester, A. Manzalini, C. Cavazzoni, G. Ricucci, U. Hartmer, M. Jaeger, G. Lehr, L. Raptis, G. Chatzillias, A. Lason, Envisaging next-generation data-centric optical networks, in proc. $3^{\text {rd }}$ International Workshop on Design of Reliable communication networks (DRCN 2001), Budapest, Hungary (October 2001), 137-144

[15] B. Ramamurthy, A. Ramakrishnan, Design of virtual private networks (VPNs) over optical wavelength division multiplexed (WDM) networks, Optical Networks Magazine, 3(1), 107121 (January/February 2002)

[16] B. Fortz, M. Thorup, Optimizing OSPF/IS--IS weights in a changing world, IEEE Journal on Selected Areas in Communications, 20(4), 756-767 (May 2002), doi: 10.1109/JSAC.2002.1003042

[17] A. Bley, M. Grötschel, R. Wessäly, Design of Broadband Virtual Private Networks: Model and Heuristics for the B-WiN, DIMACS Series in Discrete Mathematics and Theoretical Computer Science, 53, 1-16 (1998)

[18] A. Nucci, B. Schroeder, S. Bhattacharyya, N. Taft, C. Diot, IGP Link Weight Assignment for Transient Link Failures, Sprint ATL Technical Report TR02-ATL071000

[19] L.S. Buriol, P.M. França, M.G.C. Resende, M. Thorup, Network design for OSPF routing, in proc. Mathematical Programming in Rio, Búzios, Rio de Janeiro, Brazil (2003), 40-44

[20] A. Jüttnera, Á. Szentesia, J. Harmatosa, M. Piórob, On Solvability of an OSPF Routing Problem, Ericsson Traffic Laboratory, Budapest, Hungarya, Dept. of Communication Systems, Lund Institute of Technology, Sweden

[21] D. Bienstock, S. Chopra, O. Gunluk, C.-Y. Tsai, Minimum cost capacity installation for multi-commodity network flows, Journal of Mathematical Programming, 81(2), 177-199 (April 1998), doi: 10.1007/BF01581104 
[22] R.R. Iraschko, M. MacGregor, W.D. Grover, Optimal capacity placement for path restoration in STM or ATM mesh survivable networks, IEEE/ACM Transactions on Networking, 6(3) 325-336 (June 1998), doi: 10.1109/90.700896 